\title{
The effect of violent star formation on the state of the molecular gas in M 82
}

\author{
A. Weiß ${ }^{1}$, N. Neininger ${ }^{1}$, S. Hüttemeister ${ }^{1}$, and U. Klein ${ }^{1}$ \\ Radioastronomisches Institut der Universität Bonn (RAIUB), Auf dem Hügel 71, 53121 Bonn, Germany
}

Received 27 July 2000 / Accepted 11 October 2000

\begin{abstract}
We present the results of a high angular resolution, multi-transition analysis of the molecular gas in $\mathrm{M} 82$. The analysis is based on the two lowest transitions of ${ }^{12} \mathrm{CO}$ and the ground transition of the rare isotopes ${ }^{13} \mathrm{CO}$ and $\mathrm{C}^{18} \mathrm{O}$ measured with the PdBI, the BIMA array and the IRAM $30 \mathrm{~m}$ telescope. In order to address the question of how the intrinsic molecular cloud properties are influenced by massive star formation we have carried out radiative transfer calculations based on the observed $\mathrm{CO}$ line ratios. The calculations suggest that the kinetic temperature of the molecular gas is high in regions with strong star formation and drops towards the outer molecular lobes with less ongoing star formation. The location of the highest kinetic temperature is coincident with that of the mid infrared (MIR) peaks which trace emission from hot dust. The hot gas is associated with low $\mathrm{H}_{2}$ densities while the cold gas in the outer molecular lobes has high $\mathrm{H}_{2}$ densities. We find that $\mathrm{CO}$ intensities do not trace $\mathrm{H}_{2}$ column densities well. Most of the molecular gas is distributed in a double-lobed distribution which surrounds the starburst. A detailed analysis of the conversion factor from $\mathrm{CO}$ intensity to $\mathrm{H}_{2}$ column density shows that $X_{\mathrm{CO}}$ depends on the excitation conditions. We find $X_{\mathrm{CO}} \sim T_{\mathrm{kin}}^{-1} n\left(\mathrm{H}_{2}\right)^{1 / 2}$, as expected for virialized clouds.
\end{abstract}

Key words. ISM: evolution - ISM: molecules - ISM: structure - galaxies: individual: M 82 - galaxies: ISM galaxies: starburst

\section{Introduction}

M 82 is regarded as the archetypical starburst galaxy (Rieke et al. 1980). Its distance of only 3.9 Mpc (Sakai \& Madore 1999) makes M 82 an excellent laboratory for studying the relevant physical processes connected with starburst activity in detail. The central few hundred parsecs of this galaxy are heavily obscured by dust and gas which hides the central starburst region against direct observations at optical wavelengths. Evidence for the strong star-forming activity in the central region comes from radio (e.g. Kronberg et al. 1985; Wills et al. 1999) and infrared observations (e.g. Telesco \& Gezari 1992) and also from the prominent bipolar outflow visible in $\mathrm{H} \alpha$ (e.g. Bland \& Tully 1988; McKeith et al. 1995; Shopbell \& Bland-Hawthorn 1998) and X-rays (e.g. Bregman et al. 1995). The massive star formation (SF) is believed to be fueled by the large amount of molecular gas which is present in the center of M 82 .

Send offprint requests to: A. Weiß, e-mail: aweiss@astro.uni-bonn.de
On the other hand, SF affects the distribution, kinematics and physical conditions of the surrounding interstellar medium (ISM). Early studies of the distribution of the molecular gas in M 82 unveiled a double-lobed circumnuclear distribution of $\mathrm{CO}$ which was interpreted as a molecular torus with a depletion of molecular gas in the central region (Nakai et al. 1987). More recent highresolution studies by Shen \& Lo (1995) using BIMA and Neininger et al. (1998) using the IRAM interferometer at Plateau de Bure (PdBI) showed a third molecular peak $65 \mathrm{pc}$ west of the $2.2 \mu \mathrm{m}$ nucleus (Dietz et al. 1986). Using these high-resolution CO maps Weiß et al. (1999) identified an expanding superbubble in the molecular gas of M 82 which links the triple peak CO distribution and its disturbed velocity field to the prominent outflow visible in $\mathrm{H} \alpha$ and $\mathrm{X}$-rays.

Multi-transition analyses of molecular emission lines $(\mathrm{CO}, \mathrm{CS}, \mathrm{HCN})$ showed that the starburst also affects the physical conditions of the molecular gas (Wild et al. 1992; Henkel \& Bally 1985; Brouillet \& Schilke 1993). A large fraction of the molecular gas is concentrated in warm $\left(T_{\text {kin }}=50 \mathrm{~K}\right)$ and dense $\left(n\left(\mathrm{H}_{2}\right)=10^{4} \mathrm{~cm}^{-3}\right)$ clouds 
(Wild et al. 1992; Güsten et al. 1993). In a recent study Mao et al. (2000) analyzed CO $\mathrm{mm}$ and sub-mm emission lines in M 82 to investigate the physical properties of the molecular clouds. They conclude that the bulk of CO emission arises from photon-dominated regions (PDRs) while tracers of high-density gas like CS and HCN are less affected by the strong UV radiation from massive stars.

Even though these studies already provided a good global picture of the physical conditions of the molecular gas in M 82, no detailed high-resolution study exists so far that allows to resolve variations of the excitation conditions of the molecular gas over the central part of M 82. In this paper we present the results of a high angular resolution, multi-transition $\mathrm{CO}$ analysis and compare the intrinsic gas properties with observations of high-level star formation. In Sect. 2 we briefly summarize our observations and the data reduction. In Sect. 3 we describe the main results including a description of the CO morphology and kinematics, the $\mathrm{CO}$ line ratios, results from the $\mathrm{LVG}$ calculation and on $X_{\mathrm{CO}}$. In Sect. 4 we compare our results to previous studies. Section 5 summarizes our conclusions.

\section{Observations}

\subsection{PdBI observations}

We have used the PdBI to observe the ${ }^{12} \mathrm{CO}(J=2 \rightarrow 1)$ $\left(\nu_{\mathrm{obs}}=230.361 \mathrm{GHz}\right)$ and $\mathrm{C}^{18} \mathrm{O}(J=1 \rightarrow 0)$ $\left(\nu_{\mathrm{obs}}=109.698 \mathrm{GHz}\right)$ emission lines in the central region of M 82. The observations were carried out in April 1997. Due to the dual frequency setup of the PdBI we were able to observe both emission lines simultaneously. The observations were carried out in mosaic mode with seven pointings covering the central $\mathrm{kpc}$ of $\mathrm{M} 82$. The central pointing was centered on the $2.2 \mu \mathrm{m}$ nucleus at $\alpha=09^{\mathrm{h}} 55^{\mathrm{m}} 51^{\mathrm{s}} .94, \delta=69^{\circ} 40^{\prime} 47.14^{\prime \prime} \quad(\mathrm{J} 2000.0)$ (Dietz et al. 1986). The other pointings were shifted with respect to the central position by $(\alpha, \delta)=\left(-30^{\prime \prime},-8^{\prime \prime}\right)$, $\left(-20^{\prime \prime},-6^{\prime \prime}\right),\left(-10^{\prime \prime},-4^{\prime \prime}\right),\left(10^{\prime \prime}, 4^{\prime \prime}\right),\left(20^{\prime \prime}, 8^{\prime \prime}\right),\left(30^{\prime \prime}, 10^{\prime \prime}\right)$ which ensured sufficient overlap of the observed fields at $230 \mathrm{GHz}$. The primary beam of the PdBI is $22^{\prime \prime}$ and $45^{\prime \prime}$ at $230 \mathrm{GHz}$ and $109 \mathrm{GHz}$ respectively. The observations were carried out in the DC2 antenna configuration with baselines ranging from $24 \mathrm{~m}$ to $176 \mathrm{~m}$ leading to a synthesized beam of $3.8^{\prime \prime} \times 3.5^{\prime \prime}$ at $109 \mathrm{GHz}$ and $1.5^{\prime \prime} \times 1.4^{\prime \prime}$ at $230 \mathrm{GHz}$.

The $\mathrm{C}^{18} \mathrm{O}(J=1 \rightarrow 0)$ data were recorded using two correlator units leading to a total bandwidth of $780 \mathrm{~km} \mathrm{~s}^{-1}$ with $6.83 \mathrm{kms}^{-1}$ resolution. For the ${ }^{12} \mathrm{CO}(J=1 \rightarrow 0)$ transition we used four correlator units which resulted in a total bandwidth of $390 \mathrm{~km} \mathrm{~s}^{-1}$ and a velocity resolution of $3.25 \mathrm{~km} \mathrm{~s}^{-1}$. The ${ }^{12} \mathrm{CO}(J=2 \rightarrow 1)$ emission line was observed in the lower sideband of the $230 \mathrm{GHz}$, the $\mathrm{C}^{18} \mathrm{O}(J=1 \rightarrow 0)$ emission line in the upper sideband of the $109 \mathrm{GHz}$ receiver. The flux and complex bandpass calibration was determined by observing
3C 273 and MWC 349. The nearby calibrator $0836+710$ was used as a secondary amplitude and phase calibrator. The seven fields were combined in a mosaic and subsequently CLEANed using the MAPPING procedure of the GILDAS software package. This yields a roughly constant sensitivity along the major axis of M 82 with an rms noise of $6 \mathrm{mJy} /$ beam at $109 \mathrm{GHz}$ and $30 \mathrm{mJy} /$ beam at $230 \mathrm{GHz}$. For both data sets the channels with $v_{\mathrm{lsr}}>385 \mathrm{~km} \mathrm{~s}^{-1}$ and $v_{\mathrm{lsr}}<30 \mathrm{~km} \mathrm{~s}^{-1}$ were used to generate a continuum map at $109 \mathrm{GHz}$ and $230 \mathrm{GHz}$. The continuum emission was subtracted from both emission line data cubes.

\subsection{IRAM $30 \mathrm{~m}$ telescope observations}

In addition to the high-resolution $\mathrm{CO}$ data we observed the ${ }^{12} \mathrm{CO}(J=1 \rightarrow 0),{ }^{12} \mathrm{CO}(J=2 \rightarrow 1)$ and ${ }^{13} \mathrm{CO}(J=1 \rightarrow 0)$ emission lines with the IRAM $30 \mathrm{~m}$ telescope in on-the-fly mode. The observations covered an area of $3^{\prime} \times 3^{\prime}$ centered on the $2.2 \mu \mathrm{m}$ nucleus. The ${ }^{12} \mathrm{CO}(J=1 \rightarrow 0)$ observations were carried out in Nov. 1997. The ${ }^{12} \mathrm{CO}(J=2 \rightarrow 1)$ and ${ }^{13} \mathrm{CO}(J=1 \rightarrow 0)$ data were observed in Nov. 1997, Dec. 1998 and June 1999. For all observations we used the same observing procedure: The scanning velocity was $2^{\prime \prime} / \mathrm{s}$ and the readout sampling $1 \mathrm{~s}$ leading to a spatial separation of $2^{\prime \prime}$ between individual spectra in scanning direction. The spatial separation between individual scans was $4^{\prime \prime}$. Thus each on-the-fly map was sampled on a $2^{\prime \prime} \times 4^{\prime \prime}$ grid. For the ${ }^{12} \mathrm{CO}(J=1 \rightarrow 0)$ transition we performed two coverages, for the other two transitions we performed four coverages with perpendicular scanning directions. The combined data therefore were sampled on a $2^{\prime \prime} \times 2^{\prime \prime}$ grid. After first-order baseline subtraction the spectra were summed on a $3^{\prime \prime} \times 3^{\prime \prime}$ grid using the beam (11" at $230 \mathrm{GHz}, 22^{\prime \prime}$ at $109 \mathrm{GHz}$ ) and the rms noise level for weighting. This observing and reduction procedure guarantees a smooth data sampling and avoids artifacts in the combination with the interferometric data. The total integration time per beam was $65 \mathrm{~s}$ for the ${ }^{12} \mathrm{CO}(J=1 \rightarrow 0)$, $45 \mathrm{~s}$ for the ${ }^{12} \mathrm{CO}(J=2 \rightarrow 1)$ and $130 \mathrm{~s}$ for ${ }^{13} \mathrm{CO}(J=1 \rightarrow 0)$ transition resulting in an rms noise of $40 \mathrm{mK}, 65 \mathrm{mK}$ and $15 \mathrm{mK}$. As backends we used the autocorrelators which lead to a total bandwidth and velocity resolution of $650 \mathrm{~km} \mathrm{~s}^{-1} / 2.6 \mathrm{~km} \mathrm{~s}^{-1}$, $650 \mathrm{~km} \mathrm{~s}^{-1} / 1.3 \mathrm{~km} \mathrm{~s}^{-1}$ and $695 \mathrm{~km} \mathrm{~s}^{-1} / 2.7 \mathrm{~km} \mathrm{~s}^{-1}$ for the ${ }^{12} \mathrm{CO}(J=1 \rightarrow 0),{ }^{12} \mathrm{CO}(J=2 \rightarrow 1)$ and ${ }^{13} \mathrm{CO}(J=$ $1 \rightarrow 0)$ transitions respectively. For the conversion from $T_{\mathrm{A}}^{*}$ to $T_{\mathrm{mb}}$ we used $F_{\text {eff }} / B_{\text {eff }}=1.35$ at $115 \mathrm{GHz}$ (Guélin et al. 1995) and $F_{\text {eff }} / B_{\text {eff }}=2.05$ at $230 \mathrm{GHz}$ (Greve et al. 1998).

\subsection{Short-spacing correction}

To ensure that the interferometric line intensities do not suffer from missing flux due to extended emission we combined the interferometer and the single-dish data cubes. 
Table 1. Summary of the relevant parameters of the shortspacing correction

\begin{tabular}{lccc}
\hline & ${ }^{12} \mathrm{CO}(1-0)$ & ${ }^{12} \mathrm{CO}(2-1)$ & ${ }^{13} \mathrm{CO}(1-0)$ \\
\hline$F W H M$ & $22^{\prime \prime}$ & $11^{\prime \prime}$ & $22^{\prime \prime}$ \\
$D_{\text {eff }}$ & $28.1 \mathrm{~m}$ & $24.4 \mathrm{~m}$ & $28.1 \mathrm{~m}$ \\
$S_{\text {min }}$ & unknown & $24 \mathrm{~m}$ & $24 \mathrm{~m}$ \\
$S D\left[\frac{D_{\text {eff }}}{k \lambda}\right]$ & $<9.4$ & $<18.5$ & $<8.0$ \\
miss. flux & $20 \%$ & $60 \%$ & $35 \%$ \\
\hline
\end{tabular}

From top to bottom the parameters are the FWHM of the IRAM $30 \mathrm{~m}$ telescope beam, the corresponding effective diameter of the $30 \mathrm{~m}$ telescope, the shortest baseline in the interferometer data, the part of the $u v$-plane that has been replaced by the single-dish data and the average missing flux in the interferometer maps. Since the shortest baseline of the ${ }^{12} \mathrm{CO}(J=1 \rightarrow 0)$ observations from Shen \& Lo is not given in their paper we replaced the part of the $u v$-plane which corresponds to the effective diameter of the $30 \mathrm{~m}$ telescope.

For the combination we used a method that works on the final reduced (CLEANed and corrected for primary beam attenuation) interferometer cubes. The only free parameter in this method is the choice of which part of the $u v$-plane in the interferometer cube is replaced by the single-dish values. A detailed description of the method is given in the Appendix. The parameters for the $30 \mathrm{~m}$ beam sizes, the corresponding effective diameter of the $30 \mathrm{~m}$ telescope, the shortest baseline, the replaced part of the $u v-$ plane and the missing flux of the interferometer maps are given in Table 1. All reduction steps were done using the MIRIAD software package. We applied the short-spacing correction to the ${ }^{12} \mathrm{CO}(J=1 \rightarrow 0)$ cube obtained by Shen \& Lo (1995), the ${ }^{13} \mathrm{CO}(J=1 \rightarrow 0)$ cube from Neininger et al. (1998) and to the ${ }^{12} \mathrm{CO}(J=2 \rightarrow 1)$ cube.

\section{Results}

\subsection{CO morphology and kinematics}

Figures 1 and 2 show the integrated ${ }^{12} \mathrm{CO}(J=2 \rightarrow 1)$ and $\mathrm{C}^{18} \mathrm{O}(J=1 \rightarrow 0)$ line intensities. The overall morphology of both images is very similar to the ${ }^{12} \mathrm{CO}(J=1 \rightarrow$ 0) distribution published by Shen \& Lo (1995) and the ${ }^{13} \mathrm{CO}(J=1 \rightarrow 0)$ distribution published by Neininger et al. (1998). It shows a triple peak morphology of which the two outer lobes have been interpreted as the edge of a central molecular toroid (Nakai et al. 1987; Shen \& Lo $1995)$ and a weaker central peak located $65 \mathrm{pc}$ west of the M 82's center (2.2 $\mu \mathrm{m}$ peak; Dietz et al. 1986). The two outer lobes have a projected separation of $410 \mathrm{pc}\left(26^{\prime \prime}\right)$. The separation of the central and the western molecular lobe is only about $130 \mathrm{pc}\left(8^{\prime \prime}\right)$. More diffuse CO emission is detected in the ${ }^{12} \mathrm{CO}(J=2 \rightarrow 1)$ intensity distribution east and west of the $\mathrm{CO}$ peaks and in the south-west of the galaxy. The eastern part of the $\mathrm{CO}$ distribution is significantly warped to the north. The total extent of the emission region is about $1 \mathrm{kpc}$ from east to west. With respect to M 82's center the distribution of the molecular gas is clearly displaced to the west. South of the central and western CO peak two CO spurs are detected (see Fig. 1). They extend about 100 pc below the main molecular disk and join just below the expanding molecular superbubble which is located between the central and western $\mathrm{CO}$ peak (Neininger et al. 1998; Weiß et al. 1999). At the same location hot gas emerges into the halo of M 82 (e.g. Shopbell \& Bland-Hawthorn 1998; Bregman et al. 1995) supporting the idea that the $\mathrm{CO}$ spurs indicate the walls of the superbubble.

Note that the chain of $\mathrm{CO}$ emission south of the eastern end of the ${ }^{12} \mathrm{CO}(J=2 \rightarrow 1)$ distribution is most likely not real but an artifact from the primary beam correction. The kinematic of the central $400 \mathrm{pc}$ is dominated by solid body rotation. The rotation amplitude is about $200 \mathrm{~km} \mathrm{~s}^{-1}$ ranging from $115 \mathrm{~km} \mathrm{~s}^{-1}$ at the western peak up to $320 \mathrm{~km} \mathrm{~s}^{-1}$ at the eastern peak. A pv-diagram along the major axis of $\mathrm{M} 82$ in the ${ }^{12} \mathrm{CO}(J=2 \rightarrow 1)$ transition is shown in Fig. 3. (For the corresponding diagram in the $\mathrm{C}^{18} \mathrm{O}(J=1 \rightarrow 0)$ data see Weiß et al. 1999). The $p v$ diagram is centered on the brightest supernova remnant SNR 41.9+58. The intense, velocity crowded regions at $20^{\prime \prime}, 5^{\prime \prime}$ and $-7^{\prime \prime}$ offset correspond to the western, central and eastern CO peak. Between the central and western CO peaks two velocity components at $100 \mathrm{~km} \mathrm{~s}^{-1}$ and $190 \mathrm{~km} \mathrm{~s}^{-1}$ are detected. These features have been interpreted as an expanding superbubble. The velocity of the $\mathrm{CO}$ spurs is about $140 \mathrm{~km} \mathrm{~s}^{-1}$ (see Figs. 4 and 5) which is similar to the centroid velocity of the expanding superbubble. Outside the central 400 pc the CO rotation curve flattens. The dynamical center derived from the ${ }^{12} \mathrm{CO}(J=2 \rightarrow 1)$ and $\mathrm{C}^{18} \mathrm{O}(J=1 \rightarrow 0)$ data agrees very well with the value of $\mathrm{v}_{\text {sys }}=225 \pm 10 \mathrm{~km} \mathrm{~s}^{-1}$ published by Shen \& Lo (1995) for the ${ }^{12} \mathrm{CO}(J=1 \rightarrow 0)$, and Neininger et al. (1998) for the ${ }^{13} \mathrm{CO}(J=1 \rightarrow 0)$ transition. The channel maps of the ${ }^{12} \mathrm{CO}(J=2 \rightarrow 1)$ and $\mathrm{C}^{18} \mathrm{O}(J=1 \rightarrow 0)$ line emission are presented in Figs. 4 and 5 .

\subsection{Location of the starburst with respect to $\mathrm{CO}$}

Most tracers of star formation in M 82 indicate that the highest star-forming activity is not associated with the molecular peaks, which presumably indicate the location of the reservoirs for the "fuel" for star formation, but rather takes place between the peaks. The high-resolution $12.4 \mu \mathrm{m}$ image of the central region of M 82 published by Telesco \& Gezari (1992) suggests that the young stellar clusters, which heat the dust, are located between the western molecular lobe and the $2.2 \mu \mathrm{m}$ nucleus (western mid infrared (MIR) peaks), at the central CO peak, and between the central $\mathrm{CO}$ peak and the eastern CO lobe (eastern MIR peak). A similar morphology is visible in 


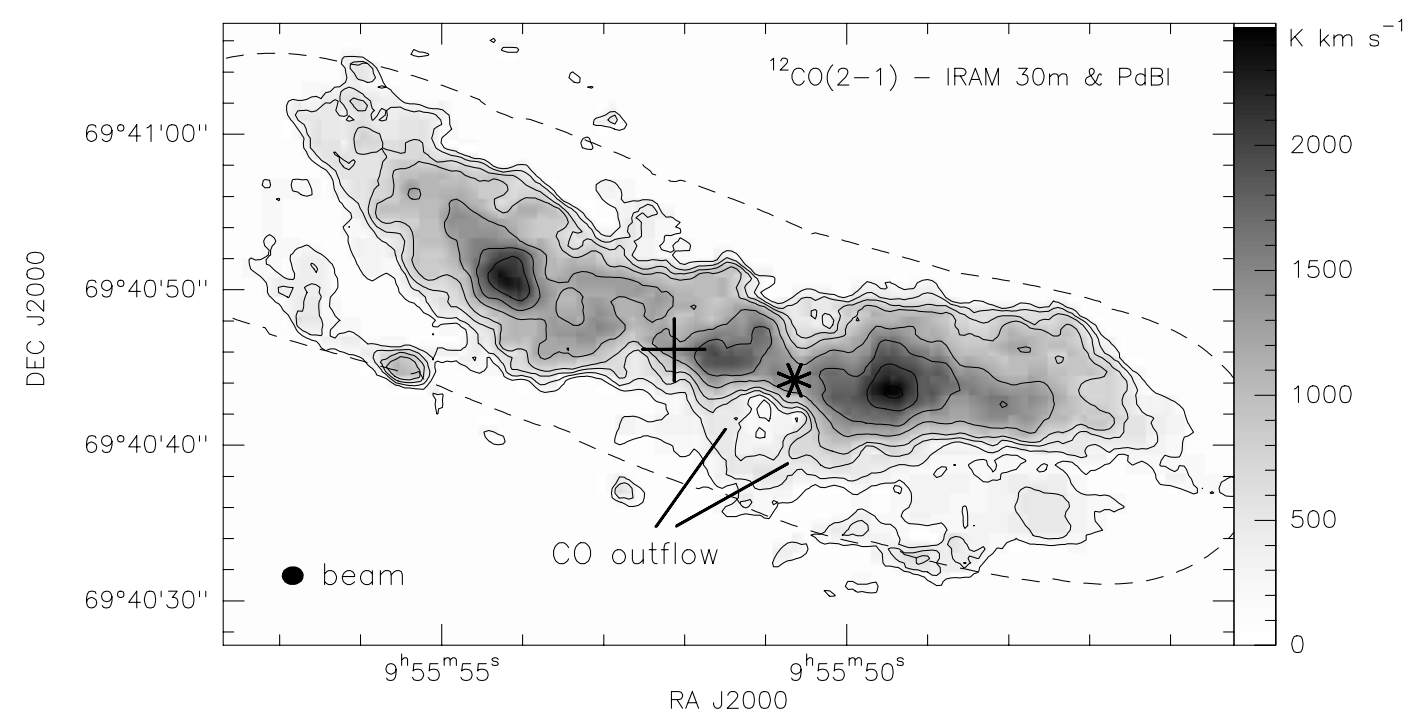

Fig. 1. Integrated ${ }^{12} \mathrm{CO}(J=2 \rightarrow 1)$ line intensity derived from the short-spacing corrected data cube. The contours correspond to $200,400,600,800,1200,1600,2000$ and $2400 \mathrm{~K} \mathrm{~km} \mathrm{~s}^{-1}$. The cross indicates the center of M $82(2.2 \mu \mathrm{m}$ peak), the star represents the location of the brightest radio continuum point source (SNR 41.9+58) in M 82 . The dotted line shows the $50 \%$ sensitivity level of the primary beam mosaic

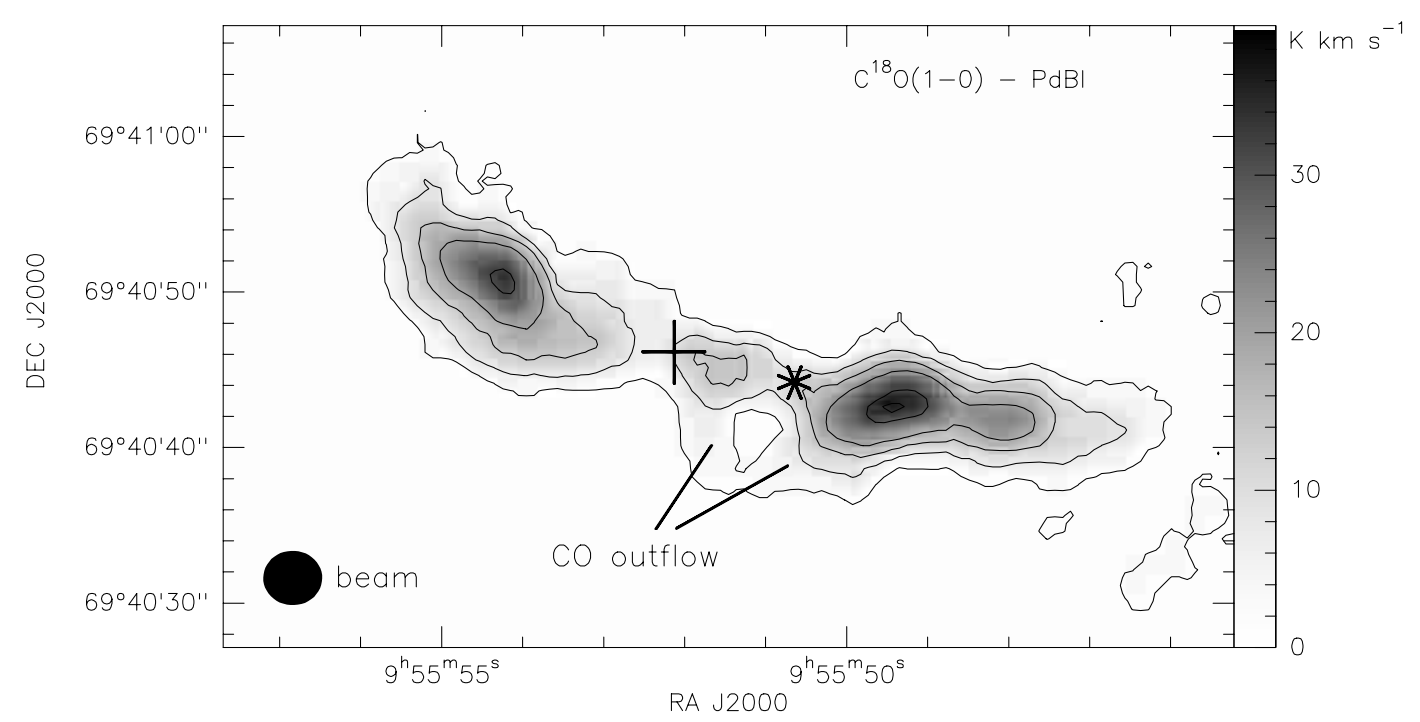

Fig. 2. Integrated $\mathrm{C}^{18} \mathrm{O}(J=1 \rightarrow 0)$ line intensity. The contours correspond to 2, 8, 14, 20, 26 and $32 \mathrm{Kkms}^{-1}$. The cross indicates the center of M $82(2.2 \mu \mathrm{m}$ peak), the star marks the location of the brightest radio continuum point source (SNR $41.9+58)$ in $\mathrm{M} 82$

the NeII line emission (Achtermann \& Lacy 1995). The radio continuum point sources, which are believed to be supernova remnants (SNR) and compact HII regions, are spread across a much wider region and seem to avoid MIR and NeII peaks (Kronberg et al. 1985). Only the strongest SNR in M 82, SNR41.9+58, appears to be related to features at other wavelengths: it is located near the center of the expanding molecular superbubble, between the central and western $\mathrm{CO}$ peak, from which hot X-ray emitting gas is released into the halo of M 82 (Weiß et al. 1999). At the same location recent radio continuum studies by
Wills et al. (1999) identified a blow-out in the form of a cone of missing $5-\mathrm{GHz}$ continuum emission. In the same study three other chimneys were identified within the central $300 \mathrm{pc}$ of M 82 . All these observations indicate that the regions of violent star formation are confined by the molecular lobes. Since no indications for high activity have been found at the $2.2 \mu \mathrm{m}$ nucleus itself, it seems that the starburst is arranged in a toroidal topology around the nucleus. 


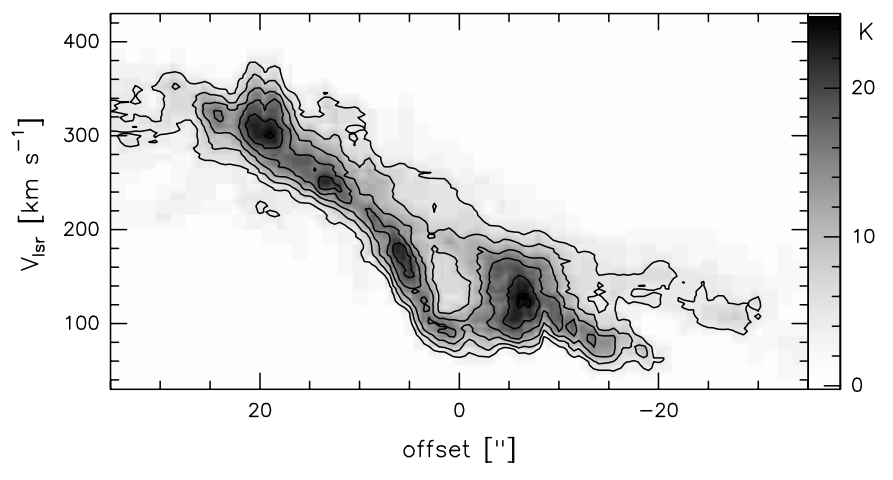

Fig. 3. A $p v$-diagram along the major axis of $M 82$ in the ${ }^{12} \mathrm{CO}(J=2 \rightarrow 1)$ transition. The contours correspond to $4,8,12,14,18$, and $22 \mathrm{~K}$. The $p v$-diagram is centered on the brightest radio continuum point source SNR $41.9+58$ (see Figs. 1 and 2). The velocity crowded regimes at $20^{\prime \prime}, 5^{\prime \prime}$ and $-7^{\prime \prime}$ offset correspond to the western, central and eastern CO peak

\subsection{CO line ratios}

To calculate the line ratios properly we used the shortspacing corrected ${ }^{12} \mathrm{CO}(J=1 \rightarrow 0),{ }^{12} \mathrm{CO}(J=2 \rightarrow 1)$ and ${ }^{13} \mathrm{CO}(J=1 \rightarrow 0)$ data cubes. Note that the missing flux in the pure interferometric maps can be as high as $60 \%$ (see Table 1). Therefore the short-spacing correction is vital to derive proper line ratios. The short-spacing correction is less crucial for the peak line intensities. Here the missing flux is $10 \%-30 \%$ only. The ${ }^{12} \mathrm{CO}(J=1 \rightarrow 0)$, ${ }^{12} \mathrm{CO}(J=2 \rightarrow 1)$ and $\mathrm{C}^{18} \mathrm{O}(J=1 \rightarrow 0)$ data were smoothed to the resolution of the ${ }^{13} \mathrm{CO}(J=1 \rightarrow 0)$ observations $\left(4.2^{\prime \prime}\right)$. Since no single-dish data were obtained for the $\mathrm{C}^{18} \mathrm{O}(J=1 \rightarrow 0)$ transition we applied the missing flux factors derived from the ${ }^{13} \mathrm{CO}(J=1 \rightarrow 0)$ peak intensity distribution to the $\mathrm{C}^{18} \mathrm{O}(J=1 \rightarrow 0)$ observations. This procedure is justified because the frequency of both transitions is similar and the observations were carried out in the same configurations with the PdBI. This leads to similar $u v$-coverages for both observations. Furthermore the morphology in the interferometer maps is similar and both transitions are optically thin (see Sect. 3.4). To take the remaining uncertainties into account we assumed an error of $50 \%$ for the $\mathrm{C}^{18} \mathrm{O}(J=1 \rightarrow 0)$ line intensities. The line ratios were calculated at 19 positions across the CO distribution of M 82. The spacing between individual positions is about $4^{\prime \prime}$. The analyzed positions are marked by the crosses in Fig. 8. The circles indicate the $F W H M$ of $4.2^{\prime \prime}$ used in the study. The positions include all molecular peaks, the $2.2 \mu \mathrm{m}$ nucleus, the MIR peaks, the CO spurs and the diffuse emission in the outer regions of M 82. For clarity the positions have been labeled 1 to 16 from east to west. Positions 17 to 19 correspond to positions on the CO outflow (see Fig. 8). The line ratios at the analyzed positions are summarized in Table 2. Errors include $10 \%$ uncertainty of the flux calibrators, errors of the amplitude calibration (typically about 10\%) and statistical errors. Our high-resolution line ratios for ${ }^{12} \mathrm{CO}$ and ${ }^{13} \mathrm{CO}$ differ slightly from values derived from single dish observations by Mao et al. (2000). But our data confirms that ${ }^{12} \mathrm{CO}(J=2 \rightarrow 1) /{ }^{12} \mathrm{CO}(J=1 \rightarrow 0)$ ratios larger than 1.8 (e.g. Knapp et al. 1980; Olofsson \& Rydbeck 1984; Loiseau et al. 1990) can firmly be rejected. ${ }^{12} \mathrm{CO}(J=1 \rightarrow 0) /{ }^{13} \mathrm{CO}(J=1 \rightarrow 0)$ and ${ }^{12} \mathrm{CO}(J=1 \rightarrow 0) / \mathrm{C}^{18} \mathrm{O}(J=1 \rightarrow 0)$ line intensity ratios are about $10-20$ and $40-60$ respectively.

\subsection{Radiative transfer calculations}

The excitation conditions of the CO-emitting volume were modeled using a spherical, isothermal one-component large velocity gradient (LVG) model (Goldreich \& Kwan 1974; de Jong et al. 1975). LVG line intensities were calculated for a kinetic temperature and $\mathrm{H}_{2}$ density range from $5 \mathrm{~K}$ to $200 \mathrm{~K}$ by $5 \mathrm{~K}$ and $\log n\left(\mathrm{H}_{2}\right)$ from 1.8 to 5.0 by 0.2 respectively. In addition, we varied the $\mathrm{CO}$ abundance relative to $\mathrm{H}_{2}$, [CO], per velocity gradient and the fractional ${ }^{13} \mathrm{CO}$ and $\mathrm{C}^{18} \mathrm{O}$ abundances $([\mathrm{CO}] / \operatorname{grad}(V)$ : $110^{-5}$ to $210^{-4}$ by $110^{-6}$; [CO]/[ $\left.{ }^{13} \mathrm{CO}\right]: 30$ to 100 by 5 ; $[\mathrm{CO}] /\left[\mathrm{C}^{18} \mathrm{O}\right]: 100$ to 300 by 20$)$. For the comparison between the observed peak intensity ratios (Table 2, Cols. 3, $5,7)$ and the predicted LVG ratios we used a $\chi^{2}$ test. To account for the absolute intensities across the disk of M 82 we also fitted the ${ }^{12} \mathrm{CO}(J=2 \rightarrow 1)$ intensity at each position by varying the beam filling from 0.1 to 0.9 by 0.1 . The "best" solutions are shown for positions 3 and 9 in Figs. 6 and 7. Position 3 on the western $\mathrm{CO}$ lobe is an example for a solution with low kinetic temperatures and high $\mathrm{H}_{2}$ densities; position 9 on the brightest MIR peak is representative for solutions with high kinetic temperatures and low $\mathrm{H}_{2}$ densities.

The observed line ratios and ${ }^{12} \mathrm{CO}(J=2 \rightarrow 1)$ intensities can be modeled within the errors at all positions. The fit agrees very well with the data at positions where ${ }^{12} \mathrm{CO}(J=2 \rightarrow 1) /{ }^{12} \mathrm{CO}(J=1 \rightarrow 0)$ is less than 1.2. At position 6 (eastern MIR peak) we do not find any intersection for all observed line ratios in the $\mathrm{H}_{2}$ density and kinetic temperature plane. For a more detailed discussion see Sect. 4.1. The best agreement with the observed line ratios and absolute intensities is found for a beam filling of 0.4. Positions 6 and 7 at the eastern MIR peak (Telesco \& Gezari 1992) and positions 18 and 19 at the CO outflow require a somewhat lower beam filling of 0.2 and 0.3 respectively.

The LVG parameters of the "best-fit" across the major axis of M82 are shown in Figs. 8a-d. The CO abundance relative to $\mathrm{H}_{2}$ per velocity gradient $([\mathrm{CO}] / \operatorname{grad}(V))$ varies between $110^{-5} \mathrm{pc} / \mathrm{km} \mathrm{s}^{-1}$ and $710^{-5} \mathrm{pc} / \mathrm{km} \mathrm{s}^{-1}$. Assuming $\operatorname{grad}(V) \approx 1 \mathrm{~km} \mathrm{~s}^{-1} \mathrm{pc}^{-1}$, as suggested by comparing the linewidth with the linear extent of the region, this corresponds to $\mathrm{CO}$ abundances in the range of $[\mathrm{CO}] \approx 10^{-5}-710^{-5}$. Similar values have been determined in the Orion region (Blake et al. 1987) and were suggested by chemical models (Farquhar et al. 1994). 
Table 2. CO line ratios in M 82 at $4^{\prime \prime}$ resolution. The offsets are given relative to $\alpha=09^{\mathrm{h}} 55^{\mathrm{m}} 51^{\mathrm{s}} .94, \delta=69^{\circ} 40^{\prime} 47.14^{\prime \prime}$ (J2000.0). For Cols. 8 \& 9 we have corrected the $\mathrm{C}^{18} \mathrm{O}$ intensities with the missing flux factor determined from the ${ }^{13} \mathrm{CO}$ data. The corresponding percentage is given in Col. 10. Errors include 10\% uncertainty of the flux calibrators, errors of the amplitude calibration (typically about $10 \%$ ) and statistical errors. For the $\mathrm{C}^{18} \mathrm{O}$ intensities we assumed an error of $50 \%$ due to the unknown missing flux

\begin{tabular}{|c|c|c|c|c|c|c|c|c|c|}
\hline & $\begin{array}{l}\Delta \alpha \\
{\left[{ }^{\prime \prime}\right]}\end{array}$ & $\begin{array}{l}\Delta \delta \\
{\left[{ }^{\prime \prime}\right]}\end{array}$ & $\frac{I(\mathrm{CO}(2-1))}{I(\mathrm{CO}(1-0))}$ & $\frac{T(\mathrm{CO}(2-1))}{T(\mathrm{CO}(1-0))}$ & $\frac{I(\mathrm{CO}(1-0))}{I(13 \mathrm{CO}(1-0))}$ & $\frac{T(\mathrm{CO}(1-0))}{T\left({ }^{13} \mathrm{CO}(1-0)\right)}$ & $\frac{I(\mathrm{CO}(1-0))}{I\left(\mathrm{C}^{18} \mathrm{O}(1-0)\right)}$ & $\frac{T(\mathrm{CO}(1-0))}{T\left(\mathrm{C}^{18} \mathrm{O}(1-0)\right)}$ & $\begin{array}{c}\mathrm{MF}^{13} \mathrm{CO}^{1} \\
{[\%]}\end{array}$ \\
\hline 1 & 16.5 & 7.5 & $1.16 \pm 0.3$ & $1.02 \pm 0.3$ & $15.2 \pm 4.9$ & $19.2 \pm 5.7$ & $44 \pm 24$ & $66 \pm 35$ & 23.5 \\
\hline 2 & 14.5 & 5.0 & $1.17 \pm 0.3$ & $0.96 \pm 0.3$ & $12.7 \pm 3.9$ & $15.4 \pm 4.9$ & $38 \pm 21$ & $45 \pm 24$ & 10.3 \\
\hline 3 & 11.5 & 3.0 & $1.12 \pm 0.3$ & $0.98 \pm 0.3$ & $11.5 \pm 3.4$ & $12.2 \pm 3.7$ & $40 \pm 21$ & $44 \pm 24$ & 8.0 \\
\hline 4 & 9.5 & 1.0 & $1.19 \pm 0.3$ & $1.09 \pm 0.3$ & $12.0 \pm 3.6$ & $13.0 \pm 4.1$ & $39 \pm 21$ & $43 \pm 23$ & 7.0 \\
\hline 5 & 6.5 & 0.5 & $1.34 \pm 0.4$ & $1.03 \pm 0.3$ & $14.2 \pm 4.4$ & $12.8 \pm 4.2$ & $37 \pm 20$ & $40 \pm 22$ & 9.0 \\
\hline 6 & 4.0 & 0.5 & $1.42 \pm 0.4$ & $1.28 \pm 0.4$ & $15.0 \pm 4.8$ & $12.6 \pm 4.1$ & $33 \pm 18$ & $37 \pm 20$ & 12.4 \\
\hline 7 & 2.0 & -0.5 & $1.39 \pm 0.4$ & $1.26 \pm 0.4$ & $17.3 \pm 5.7$ & $16.5 \pm 6.1$ & $37 \pm 20$ & $61 \pm 33$ & 10.3 \\
\hline 8 & -1.0 & -1.5 & $1.06 \pm 0.3$ & $1.01 \pm 0.3$ & $25.9 \pm 8.5$ & $20.7 \pm 8.0$ & $36 \pm 20$ & $56 \pm 30$ & 13.0 \\
\hline 9 & -4.0 & -2.0 & $0.98 \pm 0.3$ & $0.98 \pm 0.3$ & $21.3 \pm 6.6$ & $22.1 \pm 8.4$ & $53 \pm 28$ & $56 \pm 30$ & 15.6 \\
\hline 10 & -6.5 & -3.0 & $1.07 \pm 0.3$ & $0.95 \pm 0.3$ & $25.4 \pm 8.4$ & $17.0 \pm 6.0$ & $41 \pm 22$ & $45 \pm 24$ & 12.9 \\
\hline 11 & -10.0 & -4.0 & $1.12 \pm 0.3$ & $1.08 \pm 0.3$ & $13.2 \pm 3.9$ & $11.7 \pm 3.8$ & $45 \pm 24$ & $29 \pm 15$ & 14.0 \\
\hline 12 & -14.0 & -4.0 & $1.00 \pm 0.3$ & $0.98 \pm 0.3$ & $15.3 \pm 4.5$ & $11.5 \pm 3.5$ & $48 \pm 26$ & $36 \pm 20$ & 4.3 \\
\hline 13 & -17.5 & -4.0 & $1.14 \pm 0.3$ & $1.02 \pm 0.3$ & $14.6 \pm 4.4$ & $10.9 \pm 3.3$ & $48 \pm 26$ & $39 \pm 21$ & 6.6 \\
\hline 14 & -20.5 & -4.5 & $1.05 \pm 0.3$ & $0.95 \pm 0.3$ & $14.3 \pm 4.3$ & $11.1 \pm 3.6$ & $49 \pm 27$ & $39 \pm 21$ & 4.1 \\
\hline 15 & -23.5 & -4.5 & $1.07 \pm 0.3$ & $1.00 \pm 0.3$ & $16.3 \pm 5.1$ & $14.7 \pm 4.7$ & $60 \pm 32$ & $48 \pm 26$ & 5.6 \\
\hline 16 & -26.5 & -4.0 & $1.14 \pm 0.3$ & $1.09 \pm 0.3$ & $14.5 \pm 4.8$ & $14.3 \pm 4.9$ & $62 \pm 34$ & $56 \pm 30$ & 6.2 \\
\hline 17 & -2.5 & -5.5 & $1.02 \pm 0.3$ & $1.07 \pm 0.3$ & $20.2 \pm 6.8$ & $21.2 \pm 8.3$ & $40 \pm 22$ & $54 \pm 29$ & 19.0 \\
\hline 18 & -3.5 & -9.0 & $1.20 \pm 0.3$ & $0.91 \pm 0.3$ & $12.0 \pm 4.1$ & $21.5 \pm 8.6$ & $29 \pm 16$ & $52 \pm 28$ & 27.4 \\
\hline 19 & -8.5 & -7.0 & $1.36 \pm 0.4$ & $1.12 \pm 0.3$ & $11.8 \pm 3.8$ & $11.6 \pm 3.9$ & $32 \pm 17$ & $33 \pm 18$ & 10.8 \\
\hline
\end{tabular}

${ }^{1}$ Missing flux determined from the ${ }^{13} \mathrm{CO}$ data.

$[\mathrm{CO}] / \operatorname{grad}(V)$ increases towards the MIR peaks which indicates higher $\mathrm{CO}$ abundances at the active star-forming regions than in the more quiescent outer regions. The fractional ${ }^{13} \mathrm{CO}$ abundance $\left[{ }^{12} \mathrm{CO}\right] /\left[{ }^{13} \mathrm{CO}\right]$ across $\mathrm{M} 82$ does not show any significant spatial variation. The mean value of all positions is $70 \pm 20$. A low fractional ${ }^{13} \mathrm{CO}$ abundance is consistent with recent radiative transfer calculations by Mao et al. (2000) and an independent chain of arguments based on $\mathrm{CN}$ and ${ }^{13} \mathrm{CN}$ measurements (Henkel et al. 1998). In contrast, the fractional $\mathrm{C}^{18} \mathrm{O}$ abundance $\left[{ }^{12} \mathrm{CO}\right] /\left[\mathrm{C}^{18} \mathrm{O}\right]$ shows a trend towards higher $\mathrm{C}^{18} \mathrm{O}$ abundances at the MIR peaks and in the outflow. While the average $\left[{ }^{12} \mathrm{CO}\right] /\left[\mathrm{C}^{18} \mathrm{O}\right]$ ratio in the quiescent regions is about 270 , it is only about 160 at position $6,11,17$ and 19 (see Fig. 8d). Note that these values suggest $\mathrm{C}^{18} \mathrm{O}$ abundances 2-3 times higher than those used by Wild et al. (1992) for their LVG calculations of CO line ratios in M 82.

The kinetic temperature is well correlated with the MIR emission and other tracers of high-level star formation. Within the prominent CO lobes with less signs of ongoing star formation, the kinetic temperature is about $50 \mathrm{~K}$. Towards the active star-forming regions we find two kinetic temperature peaks above $150 \mathrm{~K}$. These "hot-spots" coincide with the location of MIR peaks (for a comparison between the MIR emission and the CO distribution see Telesco \& Gezari 1992). Near the $2.2 \mu \mathrm{m}$ nucleus the LVG models suggest temperatures of about $75 \mathrm{~K}$. Along the $\mathrm{CO}$ outflow the temperature drops with increasing distance from the active regions. At position 17 and 19 we find temperatures above $100 \mathrm{~K}$. At position 18 ( $100 \mathrm{pc}$ distance from the plane) the kinetic temperature has dropped to $60 \mathrm{~K}$. The spatial variation of the kinetic temperature along the major axis of M 82 is shown in Fig. 8a. The corresponding diagram of the $\mathrm{H}_{2}$ density distribution is shown in Fig. 8b. Solutions are found between $n\left(\mathrm{H}_{2}\right)=10^{2.7}$ and $10^{4.2} \mathrm{~cm}^{-3}$. In general, the $\mathrm{H}_{2}$ densities are high in regions with low kinetic temperatures and vice versa. The solutions for the outer $\mathrm{CO}$ lobes suggest an $\mathrm{H}_{2}$ density about $n\left(\mathrm{H}_{2}\right)=10^{4.0} \mathrm{~cm}^{-3}$ with a tendency towards somewhat lower values at the very edge of the $\mathrm{CO}$ distribution $\left(n\left(\mathrm{H}_{2}\right)=10^{3.5} \mathrm{~cm}^{-3}\right)$. These values are in agreement with $\mathrm{H}_{2}$ densities calculated by Wild et al. (1992) and Mao et al. (2000). At the "hot-spot", low $\mathrm{H}_{2}$ densities of $n\left(\mathrm{H}_{2}\right)=10^{2.8-3.1} \mathrm{~cm}^{-3}$ are required to match the observed line ratios. $\mathrm{H}_{2}$ densities in the $\mathrm{CO}$ outflow are about $n\left(\mathrm{H}_{2}\right)=10^{3.0} \mathrm{~cm}^{-3}$. 


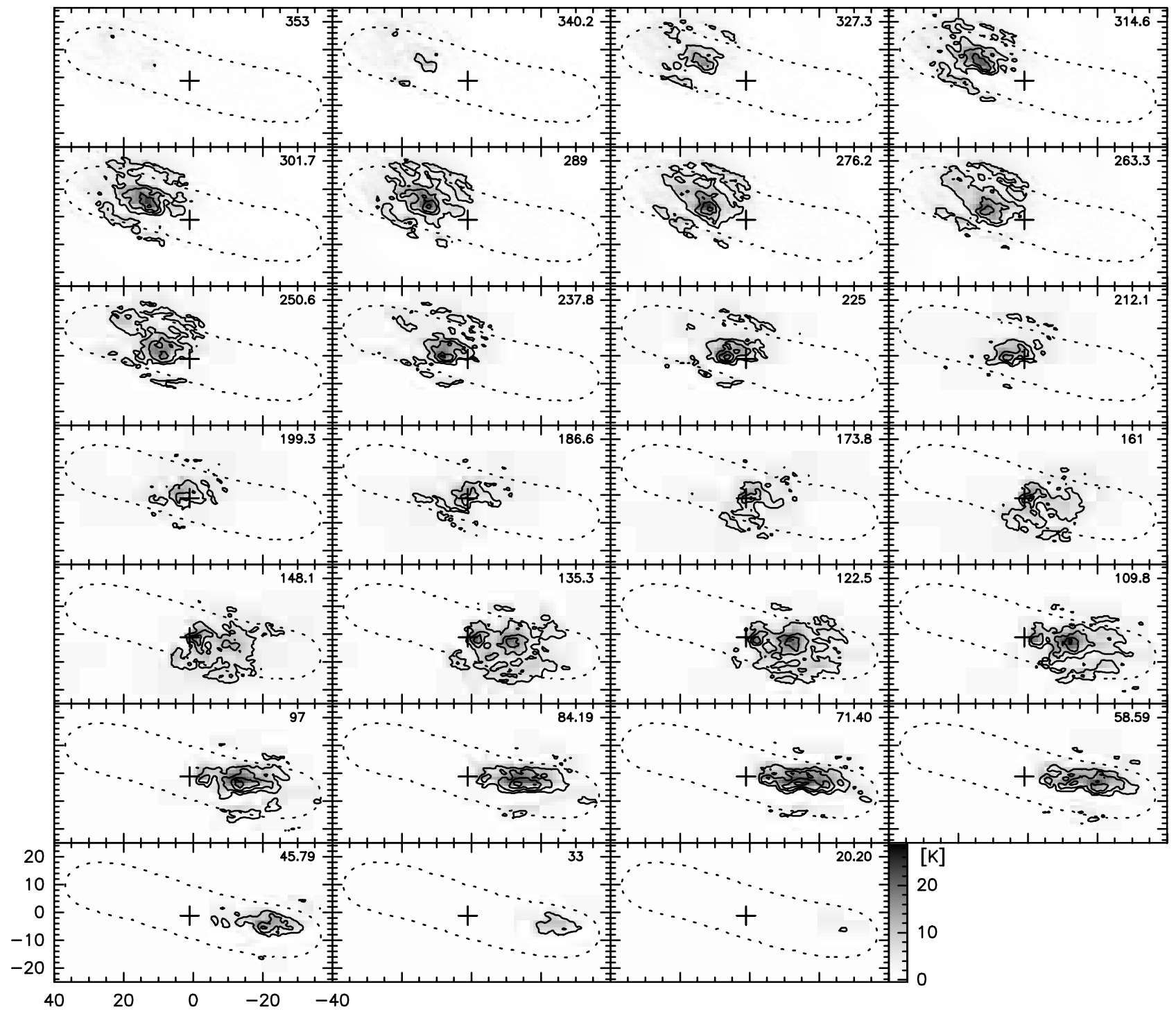

Fig. 4. ${ }^{12} \mathrm{CO}(J=2 \rightarrow 1)$ velocity channel maps of the short-spacing corrected data cube. Offsets in RA, DEC are given relative to the $2.2 \mu \mathrm{m}$ nucleus indicated by the cross in each channel map. The velocity spacing between individual maps is $12.8 \mathrm{~km} \mathrm{~s}^{-1}$, the central velocity $\left(V_{\mathrm{lsr}}\right)$ of each map is given in the top right corner in units of $\mathrm{km} \mathrm{s}^{-1}$. The intensities are corrected for attenuation of the primary beam. The contours correspond to 6, 12, 18, 24 and $30 \mathrm{~K}$. The dotted line in each channel map shows the $50 \%$ sensitivity level of the primary beam mosaic

Both the ${ }^{12} \mathrm{CO}(J=1 \rightarrow 0)$ and the ${ }^{12} \mathrm{CO}(J=2 \rightarrow 1)$ transitions are optically thick. In the cold dense regions we find an optical depth of $\tau_{12} \mathrm{CO}(J=1 \rightarrow 0)=2-5$ and $\tau_{12} \mathrm{CO}(J=2 \rightarrow 1)=7-15$. At the "hot-spots" the derived optical depths are somewhat lower and reach unity in the ${ }^{12} \mathrm{CO}(J=1 \rightarrow 0)$ transition at the eastern MIR peak (position $6 \& 7$ ). For the ground transitions of the rare isotopes ${ }^{13} \mathrm{CO}$ and $\mathrm{C}^{18} \mathrm{O}$ we find optically thin emission at all positions. Typical optical depths are $\tau_{13} \mathrm{CO}(J=1 \rightarrow 0)=$ $510^{-2}$ and $\tau_{\mathrm{C}^{18} \mathrm{O}(J=1 \rightarrow 0)}=510^{-3}$.

\subsection{Column densities and mass distribution}

For the determination of $\mathrm{CO}$ and $\mathrm{H}_{2}$ column densities at each position we used three methods:
- LVG: The column densities were derived from the CO and $\mathrm{H}_{2}$ densities, the velocity gradient and the observed line widths using $N(\mathrm{CO})=3.0810^{18} n(\mathrm{CO}) \frac{\mathrm{d} V}{\operatorname{grad}(V)}$ and $N\left(\mathrm{H}_{2}\right)=3.0810^{18} n\left(\mathrm{H}_{2}\right) \frac{\mathrm{d} V}{\operatorname{grad}(V)}$, where $\mathrm{d} V$ is the observed line width. Therefore $\frac{\mathrm{d} V}{\operatorname{grad}(V)}$ is an equivalent path length through the clouds;

- $\mathrm{LVG}_{\mathrm{PF}}$ (PF = partition function): the ${ }^{13} \mathrm{CO}$ and $\mathrm{C}^{18} \mathrm{O}$ column densities were calculated from the general relation between optical depth, excitation temperature and column density at rotation level $J: N_{J}=93.5 \frac{g_{J} \nu^{3}}{g_{(J+1)} A_{J+1, J}}(1-$ $\left.\exp \left(-4.810^{-2} \nu / T_{\mathrm{ex}}\right)\right)^{-1} \int \tau \mathrm{d} v$ where $g_{J}$ is the statistical weight of level $J$ and $A_{J+1, J}$ is the Einstein coefficient for the transition $J+1$ to $J$. $\int \tau \mathrm{d} v$ was approximated by $\int \tau \mathrm{d} v \approx 1.06 \tau \mathrm{d} V . T_{\text {ex }}$ and $\tau$ are given by the LVG code for each level. ${ }^{13} \mathrm{CO}$ and $\mathrm{C}^{18} \mathrm{O}$ column densities were 


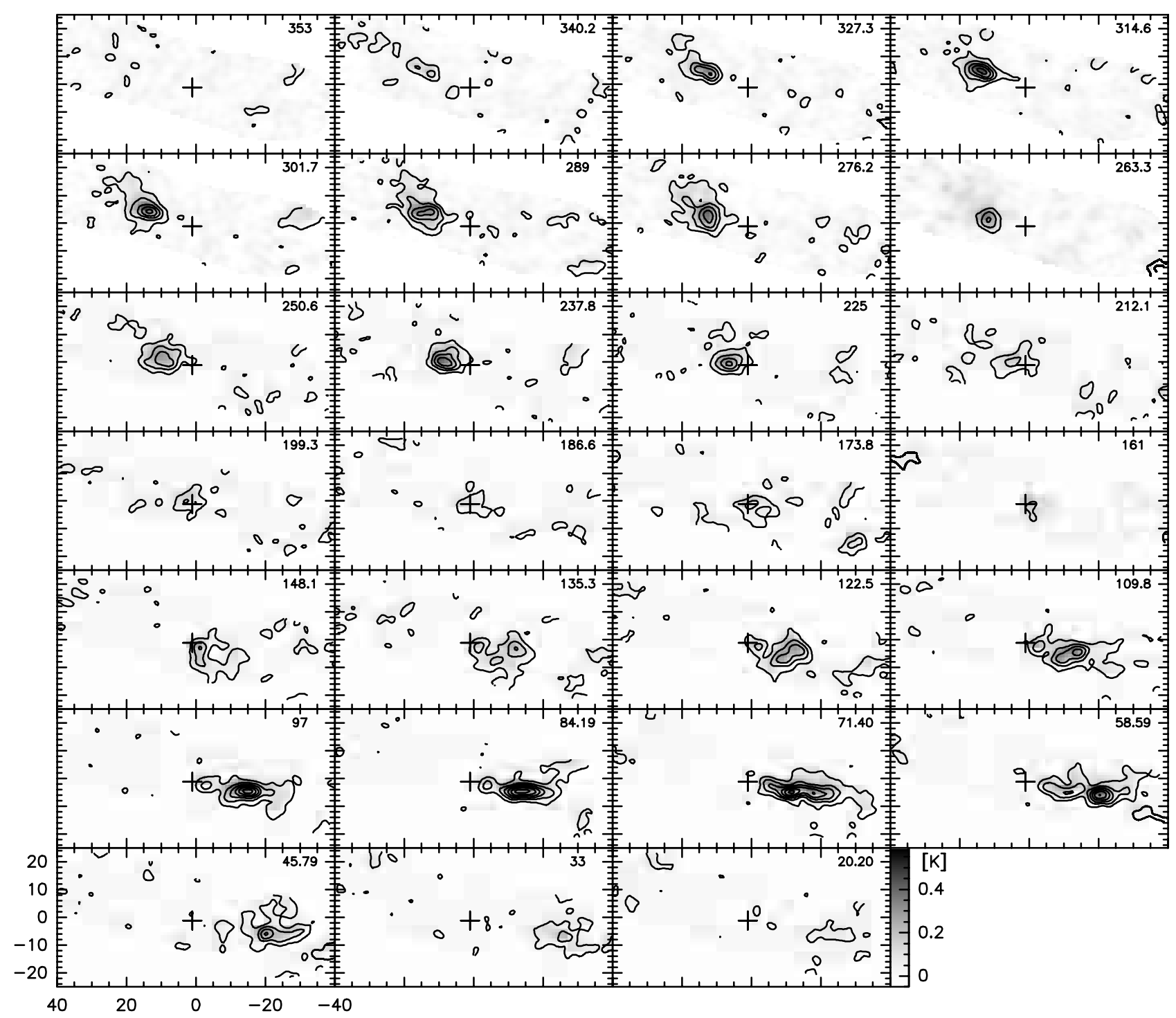

Fig. 5. $\mathrm{C}^{18} \mathrm{O}(J=1 \rightarrow 0)$ velocity channel maps of the PdBI data cube. The figure has the same layout and specifications as Fig. 4; contours correspond to $0.05,0.15,0.25,0.35,0.45$ and $0.55 \mathrm{~K}$

determined using the sum of the 6 lowest levels for each isotope. $\mathrm{H}_{2}$ and $\mathrm{CO}$ column densities were derived from the relative abundances of the rare isotopes relative to $\mathrm{H}_{2}$ and $\mathrm{CO}$;

- LTE: ${ }^{13} \mathrm{CO}$ and $\mathrm{C}^{18} \mathrm{O}$ column densities were derived using a standard LTE approach (e.g. Dickman 1978). As for the $\mathrm{LVG}_{\mathrm{PF}}$ method, $\mathrm{CO}$ and $\mathrm{H}_{2}$ column densities were derived from the abundances of the rare isotopes relative to $\mathrm{CO}$ and $\mathrm{H}_{2}$ at each position.

Column densities calculated from ${ }^{13} \mathrm{CO}$ and $\mathrm{C}^{18} \mathrm{O}$ via the LTE method match each other with less than $5 \%$ difference at each position. The same holds for the $\mathrm{LVG}_{\mathrm{PF}}$ column densities calculated from $T_{\text {ex }}$ and $\tau$ of the ${ }^{13} \mathrm{CO}$ and $\mathrm{C}^{18} \mathrm{O}$ transition. For simplicity we therefore give in the following the average between the column densities calculated from ${ }^{13} \mathrm{CO}$ and $\mathrm{C}^{18} \mathrm{O}$ via the LTE and $\mathrm{LVG}_{\mathrm{PF}}$ method.
The spatial variations of the beam-averaged $\mathrm{H}_{2}$ column density across the major axis of M 82 as calculated with the three methods is shown in Fig. 9. The spatial distribution of the $\mathrm{H}_{2}$ column densities is in good agreement for all three methods. This suggests that the low $J$ levels are almost thermalized. The largest difference between the methods is apparent at the central CO peak. While the LTE solutions suggest a local $\mathrm{H}_{2}$ column density maximum of about $N\left(\mathrm{H}_{2}\right)_{4^{\prime \prime}}=110^{23} \mathrm{~cm}^{-2}$, the peak is less prominent $\left(N\left(\mathrm{H}_{2}\right)_{4^{\prime \prime}}=510^{22} \mathrm{~cm}^{-2}\right)$ and displaced by $4^{\prime \prime}$ in the LVG and LVG $_{\mathrm{PF}}$ solution (see Fig. 9).

Nevertheless, all methods clearly show that most of the molecular gas traced by $\mathrm{CO}$ is located in the outer CO lobes. The central $300 \mathrm{pc}$ between the molecular lobes contain only about $20-30 \%$ of the molecular gas mass. Furthermore, the $\mathrm{H}_{2}$ column density distribution is clearly asymmetric with respect to the $2.2 \mu \mathrm{m} \mathrm{nu}-$ cleus. We find that the centroid of mass is located about 

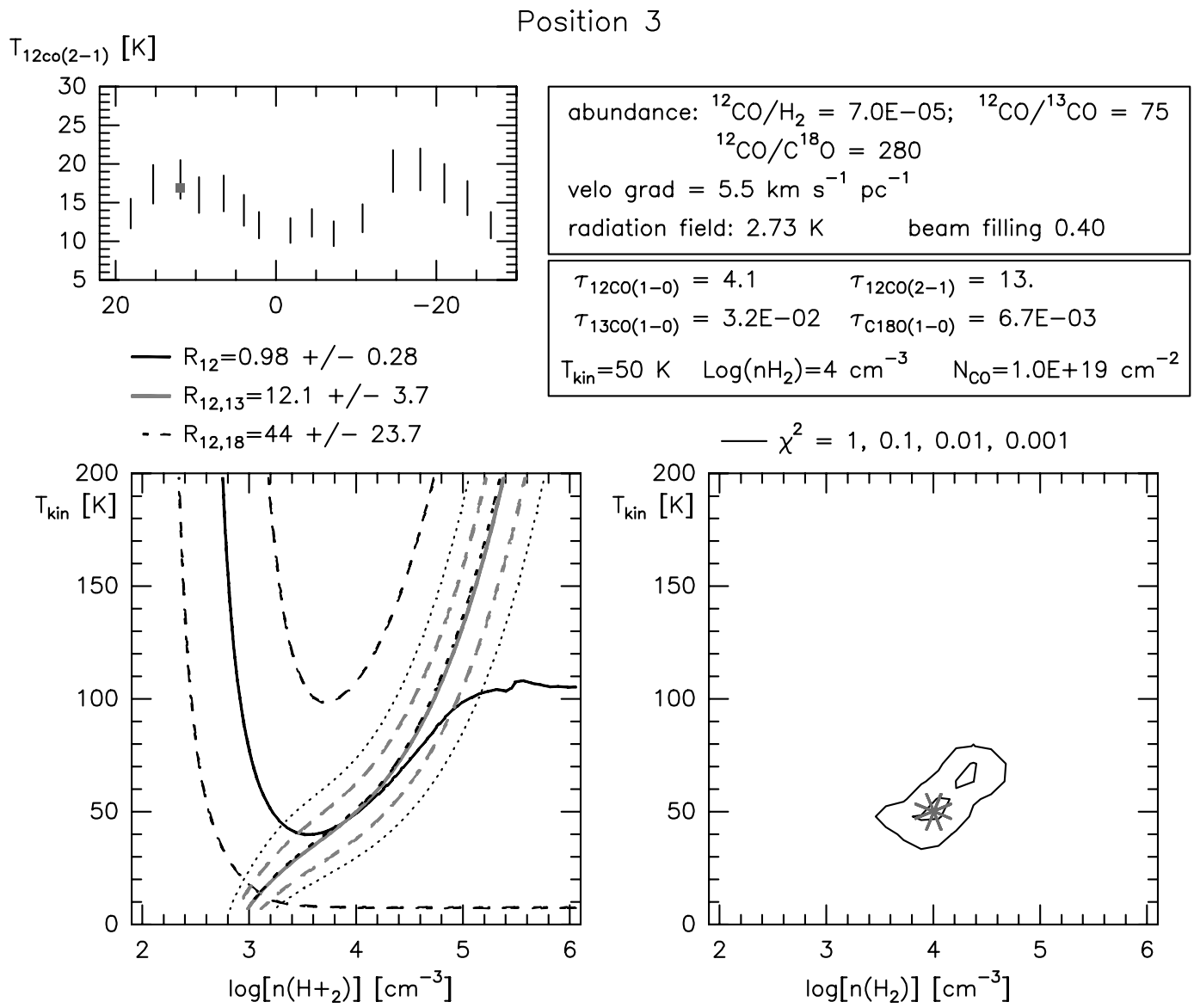

Fig. 6. "Best" LVG solution at position 3 (the eastern CO lobe). The top left diagram shows the observed ${ }^{12} \mathrm{CO}(J=2 \rightarrow 1)$ line intensities across the major axis of M 82 and the ${ }^{12} \mathrm{CO}(J=2 \rightarrow 1)$ intensity as given by the LVG-model for position 3 . The fixed LVG-parameters (abundances, velocity gradient, radiation field and beam filling) are given in the upper right box. The parameters below the LVG input parameters summarize the optical depth for each transition, the CO column density, the kinetic temperature and $\mathrm{H}_{2}$ density for the "best fit". The black and the grey solid line in the lower left diagram are the ${ }^{12} \mathrm{CO}(J=2 \rightarrow 1) /{ }^{12} \mathrm{CO}(J=1 \rightarrow 0)\left(R_{12}\right)$ and ${ }^{13} \mathrm{CO}(J=1 \rightarrow 0) /{ }^{12} \mathrm{CO}(J=1 \rightarrow 0)\left(R_{12,13}\right)$ line intensity ratio, respectively. The dashed lines indicate the observational errors for the corresponding line ratio. The $\mathrm{C}^{18} \mathrm{O}(J=1 \rightarrow 0) /{ }^{12} \mathrm{CO}(J=1 \rightarrow$ $0)\left(R_{12,18}\right)$ line intensity ratio is given by the dashed-dotted line. Its error is indicated by the dotted line. The contours in the lower right diagram are results of the $\chi^{2}$ test comparing the predicted line ratios and the ${ }^{12} \mathrm{CO}(J=2 \rightarrow 1)$ line intensity with the observed values. The star indicates the best solution in the kinetic temperature and $\mathrm{H}_{2}$ density plane

100 pc south-east of the nucleus. The location of the centroid of mass for each method is indicated by the vertical line in Fig. 9. The highest $\mathrm{H}_{2}$ column density is found at the western CO lobe (position 12). Its beam-averaged LVG column densities are $N(\mathrm{CO})_{4^{\prime \prime}}=210^{19} \mathrm{~cm}^{-2}$ and $N\left(\mathrm{H}_{2}\right)_{4^{\prime \prime}}=2.310^{23} \mathrm{~cm}^{-2}$. The corresponding cloud-averaged LVG column densities are $N(\mathrm{CO})_{\text {cloud }}=410^{19} \mathrm{~cm}^{-2}$ and $N\left(\mathrm{H}_{2}\right)_{\text {cloud }}=$ $610^{23} \mathrm{~cm}^{-2}$, respectively. The corresponding values for the eastern $\mathrm{CO}$ lobe (position 3) are $N(\mathrm{CO})_{4^{\prime \prime}}=$ $110^{19} \mathrm{~cm}^{-2}, N\left(\mathrm{H}_{2}\right)_{4^{\prime \prime}}=1.510^{23} \mathrm{~cm}^{-2}, N(\mathrm{CO})_{\text {cloud }}=$ $2.510^{19} \mathrm{~cm}^{-2}$ and $N\left(\mathrm{H}_{2}\right)_{\text {cloud }}=3.810^{23} \mathrm{~cm}^{-2}$. For an assumed line-of-sight of $350 \mathrm{pc}$ (for comparison with Mao et al. 2000) the mean molecular density in the CO lobes is $<n\left(\mathrm{H}_{2}\right)>_{4^{\prime \prime}}=140-210 \mathrm{~cm}^{-3}$. This corresponds to a volume filling factor of $f_{\mathrm{v}, 4^{\prime \prime}}=\left\langle n\left(\mathrm{H}_{2}\right)>_{4^{\prime \prime}} / n\left(\mathrm{H}_{2}\right) \approx 0.01\right.$. With $f_{\mathrm{a}, 4^{\prime \prime}}=0.4$ and a linear resolution of $65 \mathrm{pc}$ we obtain characteristic cloud sizes of $r_{\text {cloud }}=\frac{1}{2} 65 \mathrm{pc} f_{\mathrm{v}, 4^{\prime \prime}} / f_{\mathrm{a}, 4^{\prime \prime}} \approx$ 1 pc. Volume filling factors and characteristic cloud sizes do not change significantly in the central star forming regions. These values are in good agreement with PDR models published by Wolfire et al. (1990).

$\mathrm{H}_{2}$ column densities in the molecular outflow are in the range $N\left(\mathrm{H}_{2}\right)_{4^{\prime \prime}}=1.5-3.010^{22} \mathrm{~cm}^{-2}$. The total mass of the outflow is $7.210^{5} M_{\odot}(D=3.9 \mathrm{Mpc}$, Sakai \& Madore 1999).

\subsection{Conversion from $I(\mathrm{CO})$ to $N\left(\mathrm{H}_{2}\right)$ and total mass}

To derive the conversion factor from $I(\mathrm{CO})$ to $N\left(\mathrm{H}_{2}\right)$, we have compared LVG, LTE, and LVG $_{\mathrm{PF}} \mathrm{H}_{2}$ column densities with the integrated ${ }^{12} \mathrm{CO}(J=1 \rightarrow$ $0)$ intensities at $4.2^{\prime \prime}$ resolution at the analyzed positions across the central part of M 82. The variation of 


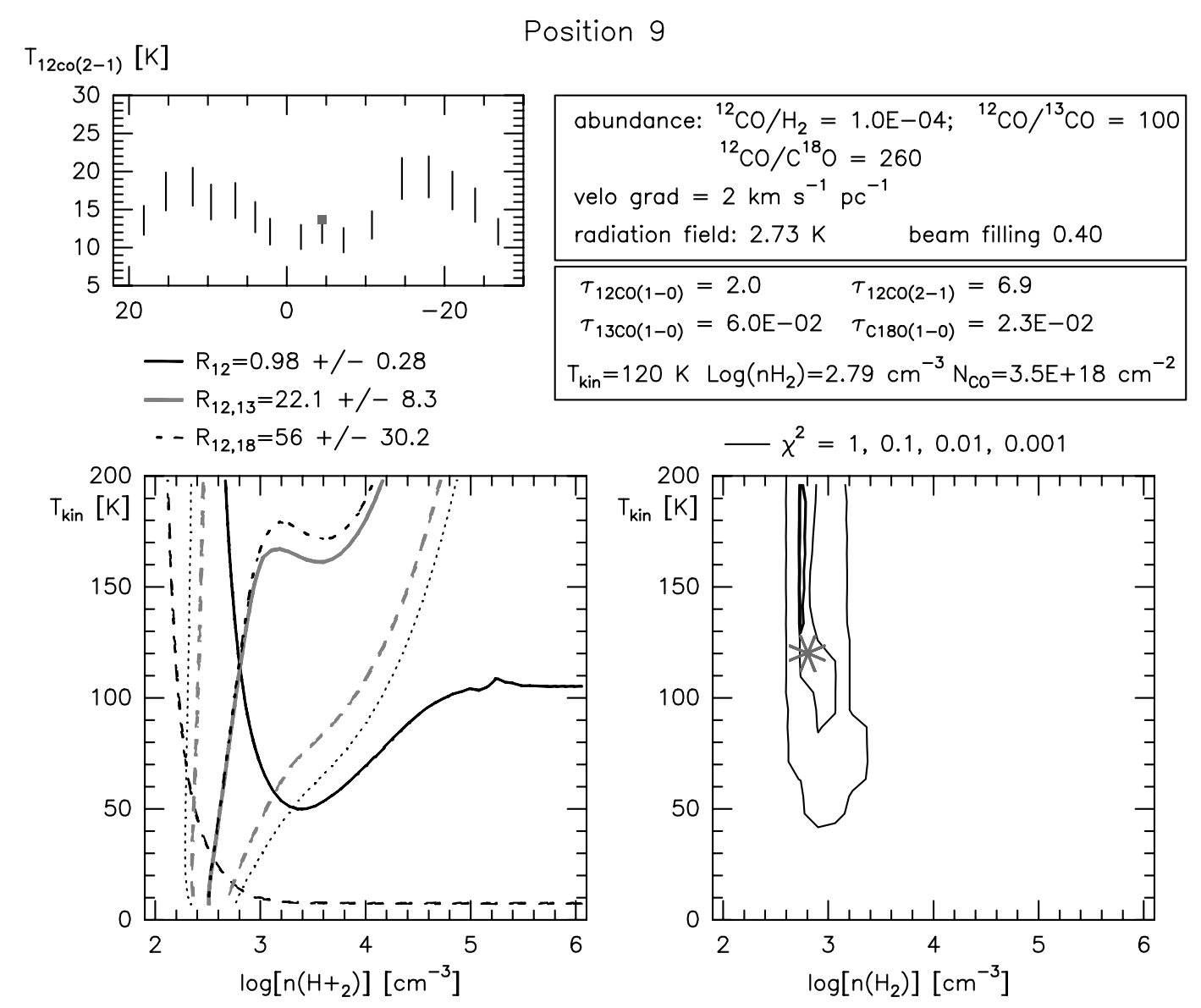

Fig. 7. "Best" LVG solution at position 9 (central MIR peak). The items and parameters are the same as in Fig. 6

the conversion factor $X_{\mathrm{CO}}=N\left(\mathrm{H}_{2}\right) / I(\mathrm{CO})$ with position is shown in Fig. 10. Note that $X_{\mathrm{CO}}$ is lower than the Galactic value of $1.610^{20} \mathrm{~cm}^{-2}\left(\mathrm{~K} \mathrm{~km} \mathrm{~s}^{-1}\right)^{-1}$ (Hunter et al. 1997) at all positions and for all methods. We find that $X_{\mathrm{CO}}$ varies across the disk of M 82 by about a factor of 5 if one considers the LTE solutions $\left(X_{\mathrm{CO}}=2.1-10.810^{19} \mathrm{~cm}^{-2}\left(\mathrm{~K} \mathrm{~km} \mathrm{~s}^{-1}\right)^{-1}\right)$ and by a factor of $8-9$ for the $L V G$ and $L G_{P F}$ solutions $\left(X_{\mathrm{CO}}=1.3-11.510^{19} \mathrm{~cm}^{-2}\left(\mathrm{~K} \mathrm{~km} \mathrm{~s}^{-1}\right)^{-1}\right.$ and $X_{\mathrm{CO}}=$ 1.5-12.2 $\left.10^{19} \mathrm{~cm}^{-2}\left(\mathrm{~K} \mathrm{~km} \mathrm{~s}^{-1}\right)^{-1}\right)$. All methods show that the lowest conversion factors are associated with the central star-forming regions where the gas is heated by UV photons from the newly formed stars and cosmic-rays from SNRs. The CO-emitting volumes at these positions have high kinetic temperatures. Towards the outer molecular lobes with higher $\mathrm{H}_{2}$ densities and lower kinetic temperatures, the conversion factor rises. This is in agreement with simple theoretical arguments that suggest that the conversion factor $X_{\mathrm{CO}}$ should be proportional to $T_{\mathrm{kin}}^{-1} n\left(\mathrm{H}_{2}\right)^{1 / 2}$ for virialized clouds (Maloney \& Black 1988). The variation of $X_{\mathrm{CO}}$ with $T_{\mathrm{kin}}^{-1} n\left(\mathrm{H}_{2}\right)^{1 / 2}$ is shown in Fig. 11. The linear correlation between $X_{\mathrm{CO}}$ and $T_{\mathrm{kin}}^{-1} n\left(\mathrm{H}_{2}\right)^{1 / 2}$ for $T_{\mathrm{kin}}^{-1} n\left(\mathrm{H}_{2}\right)^{1 / 2}>0.5$ is clearly visible. For $T_{\text {kin }}^{-1} n\left(\mathrm{H}_{2}\right)^{1 / 2}<0.5$ the scatter in the plot increases. This is in particular true for $X_{\mathrm{CO}}$ calculated under the assumption of LTE. This suggests that the gas is not close to LTE at the "hot spots". The increased scatter of $X_{\mathrm{CO}}$ calculated with the LVG and LVG might suggest that either the clouds are not virialized or that more appropriate models (like PDR models) are required to calculate the physical gas properties in the center of the starburst. For a more detailed discussion see Sect. 4.1. Nevertheless, this result not only shows that the standard Galactic $X_{\mathrm{CO}}$ factor is not appropriate for a starburst system like M 82, but that $X_{\mathrm{CO}}$ is a function of the intrinsic gas properties which strongly depend on environmental effects. This implies that spatial variations of ${ }^{12} \mathrm{CO}(J=1 \rightarrow 0)$ intensities can be due to variations of the excitation conditions of the gas rather than variations of column density. Similar results have been obtained by Wild et al. (1992) using low-resolution CO data (see also Sect. 4.3). Based on the analysis of $X_{\mathrm{CO}}$ we have calculated the "true" $\mathrm{H}_{2}$ distribution in M 82 by interpolating the changes of $X_{\mathrm{CO}}$ from the analyzed positions across the central CO distribution. Multiplication of this $X_{\mathrm{CO}}-$ map with the integrated ${ }^{12} \mathrm{CO}(J=1 \rightarrow 0)$ intensity distribution thus results in an $\mathrm{H}_{2}$ column density map. We show these maps in Fig. 12 for $X_{\mathrm{CO}}$ derived from the $\mathrm{LVG}_{\mathrm{PF}}$ (top) and LTE solutions (middle) in comparison with the $\mathrm{H}_{2}$ distribution one would derive assuming a 


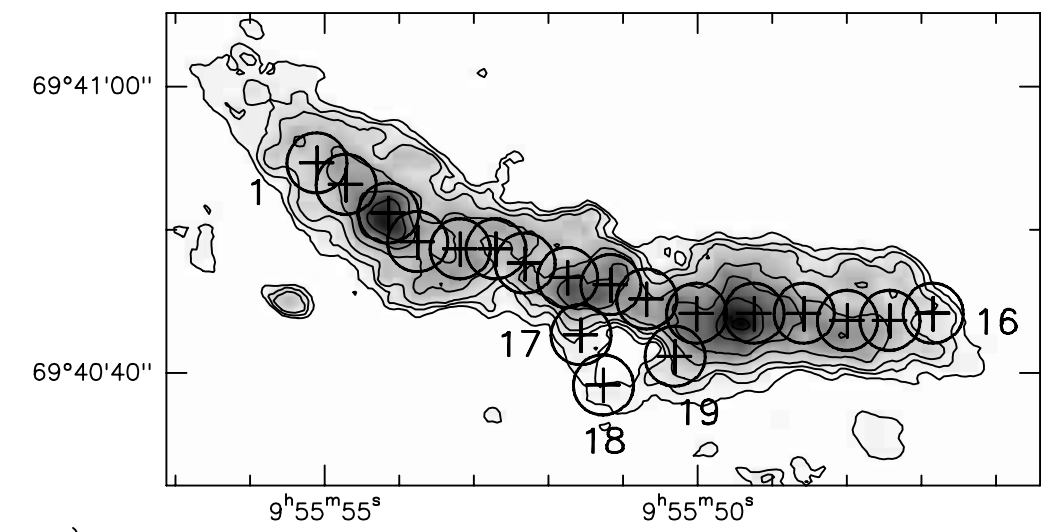

a)

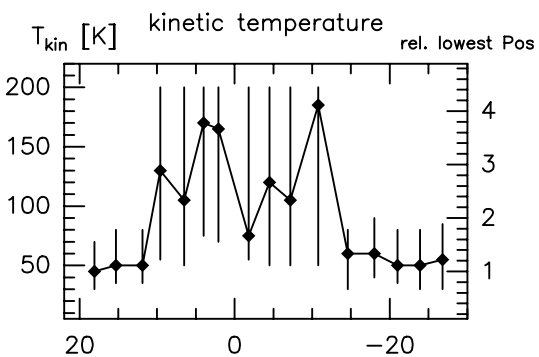

c)

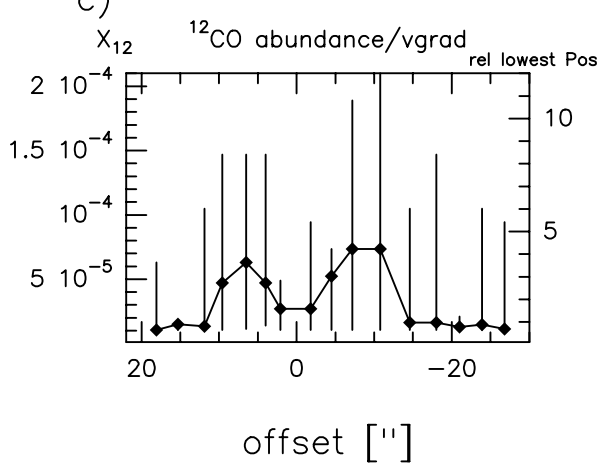

b)

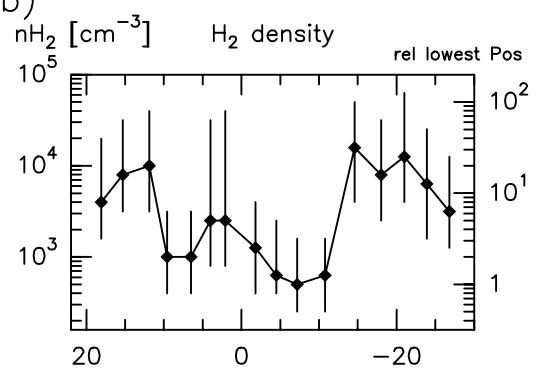

d)

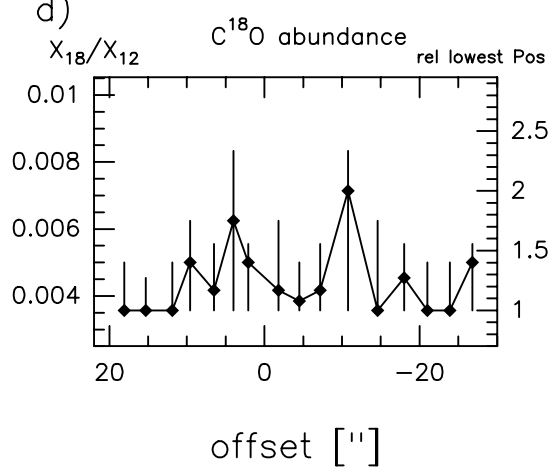

Fig. 8. LVG solutions for positions 1 to 16. Top: locations of the analyzed positions. The radii of the circles indicate the spatial resolution for which the line ratios have been determined. a) to d): spatial variations of the kinetic temperature, the $\mathrm{H}_{2}$ density, the $\mathrm{CO}$ abundance per velocity gradient and the fractional $\mathrm{C}^{18} \mathrm{O}$ abundance across the major axis of $\mathrm{M} 82$. The error bars in a) and b) correspond to the parameter range of kinetic temperatures and $\mathrm{H}_{2}$ densities for which the LVG line ratios and the ${ }^{12} \mathrm{CO}(J=2 \rightarrow 1)$ intensity is consistent with the observations within the errors. This corresponds to the area within the $\chi^{2}=1$ contour shown for position 3 and 9 in Figs. 6 and 7. The error bars in $\mathbf{c})$ and $\mathbf{d})$ correspond to the $\operatorname{range}$ for $[\mathrm{CO}] / \operatorname{grad}(V)$ and fractional $\mathrm{C}^{18} \mathrm{O}$ abundance where the $\chi^{2}$ of the corresponding fit is 50 times higher than for the "best" solution

constant, standard Galactic conversion (bottom) to illustrate the importance of detailed studies of $X_{\mathrm{CO}}$ to derive $\mathrm{H}_{2}$ column density distributions. The $\mathrm{H}_{2}$ column density maps in Fig. 12 (top and middle) indicate that the central star-forming region is surrounded by a double-lobed distribution of molecular gas, while $\mathrm{H}_{2}$ seems to be depleted in the central starburst region itself (see also Fig. 9).

The total $\mathrm{H}_{2}$ mass of the region shown in Fig. 12 is $2.310^{8} M_{\odot}$ for the $\mathrm{LVG}_{\mathrm{PF}}$ and LVG and $2.710^{8} M_{\odot}$ for the LTE solution at a distance of $D=3.9 \mathrm{Mpc}$ (Sakai \& Madore 1999). The corresponding values at $D=3.25 \mathrm{Mpc}$ (Tammann \& Sandage 1968) are 1.6 and $1.910^{8} M_{\odot}$, respectively. These values are in good agreement with estimates from $450 \mu \mathrm{m}$ dust continuum measurements
(Smith et al. 1991) and from $\mathrm{C}^{18} \mathrm{O}(2 \rightarrow 1)$ intensities (Wild et al. 1992). Therefore, the total molecular mass is 3 times lower than the mass one would derive using the standard Galactic conversion factor of $1.610^{20} \mathrm{~cm}^{-2}\left(\mathrm{~K} \mathrm{~km} \mathrm{~s}^{-1}\right)^{-1}$ $\left(4.910^{8} M_{\odot} D=3.25 \mathrm{Mpc} ; 7.110^{8} M_{\odot} D=3.9 \mathrm{Mpc}\right)$.

\section{Discussion}

\subsection{Comparison with other radiative transfer analyses}

Analyses of the physical conditions of the molecular gas in M 82 have been published by Tilanus et al. (1991), Wild et al. (1992), Güsten et al. (1993) and more recently by Mao et al. (2000) and Petitpas \& Wilson (2000) using 


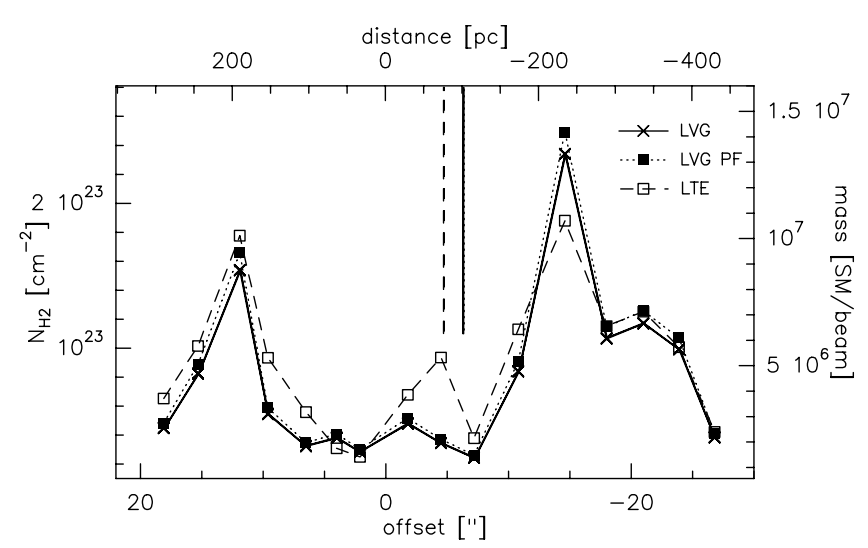

Fig. 9. Beam averaged $\mathrm{H}_{2}$ column densities across the major axis of M 82. Offsets are given relative to the center of M 82 $\left(2.2 \mu \mathrm{m}\right.$ peak). The thick solid line corresponds to the $\mathrm{H}_{2}$ column densities derived from the LVG solutions. The dashed and the dotted lines give the corresponding values for the LTE approximation and the solution calculated from the partition functions $\left(\mathrm{LVG}_{\mathrm{PF}}\right)$, respectively. The vertical lines indicate the location of the centroid of mass of the three $\mathrm{H}_{2}$ distributions. Note that the location of the centroid of mass is identical for the LVG and LVG $_{\mathrm{PF}}$ solution. The labeling of the right axes gives the $\mathrm{H}_{2}$ mass per beam in units of solar masses

single-dish CO data and other tracers of the molecular gas. The kinetic gas temperature of the $\mathrm{CO}-$ emitting gas phase derived in these studies are of order $T_{\text {kin }}=30-70 \mathrm{~K}$. $\mathrm{H}_{2}$ densities range between $n\left(\mathrm{H}_{2}\right)=10^{3}-10^{4} \mathrm{~cm}^{-3}$. Thus our solutions at the $\mathrm{CO}$ lobes and the outer parts of the $\mathrm{CO}$ distribution $\left(n\left(\mathrm{H}_{2}\right)=10^{3.5-4.2} \mathrm{~cm}^{-3}, T_{\text {kin }}=\right.$ $45-60 \mathrm{~K}$ ) are consistent with previous studies.

The situation is different for our LVG solutions within the starburst region. Kinetic gas temperatures above $150 \mathrm{~K}$ are clearly inconsistent with results published in literature so far. In the most recent analysis of the excitation conditions of the molecular gas using $\mathrm{mm}$ and sub-mm CO emission lines (up to $J=7-6$ ), Mao et al. (2000) suggested kinetic gas temperatures as high as $130 \mathrm{~K}$. However, they rejected their LVG solution because of intrinsic inconsistencies regarding almost equal area and volume filling factors derived from the one-component LVG model. This leads to characteristic cloud sizes of about $150 \mathrm{pc}$ which is inconsistent with high-resolution studies of the CO distribution in M 82 (Shen \& Lo 1995; Neininger et al. 1998 and this work). Characteristic cloud sizes derived from our analysis are only $\approx 1 \mathrm{pc}$, hence more realistic. This difference results mainly from a very low area filling factor of only $f_{\mathrm{a}, 22^{\prime \prime}}=0.04-0.07$ found by Mao et al. (2000). From the CO morphology (see Fig. 1) and $f_{\mathrm{a}, 4^{\prime \prime}}=0.4-0.2$ we would expect area filling factors of $f_{\mathrm{a}, 22^{\prime \prime}}=0.2-0.15$ at $22^{\prime \prime}$ resolution. The reason for this discrepancy remains unclear. But obviously the assumption of an isothermal gas phase used in the LVG model is more reasonable for our high spatial resolution study than for the lowresolution data used by Mao et al. (2000). Even though

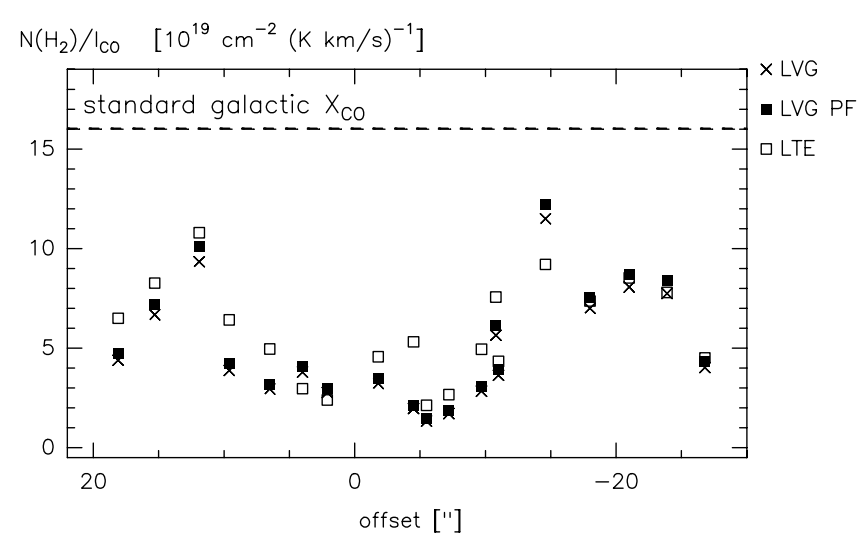

Fig. 10. Variation of $X_{\mathrm{CO}}$ across the analyzed positions in M 82. Offsets are given relative to the center of M82 (2.2 $\mu \mathrm{m}$ peak). The $\mathrm{H}_{2}$ column densities were derived using the LTE, the LVG and LVG $_{P F}$ solutions. The dotted line at $X_{\mathrm{CO}}=1.610^{20} \mathrm{~cm}^{-2}\left(\mathrm{~K} \mathrm{~km} \mathrm{~s}^{-1}\right)^{-1}$ corresponds to the standard Galactic conversion factor

our LVG analysis does not lead to internal inconsistencies we also find that the observed line ratios are difficult to reproduce with the one-component LVG model at the "hot spots". This is in particular true for positions 6,7 and 11 (eastern MIR peak and expanding superbubble) where no intersection of all observed line ratios (disregarding the errors of the observations) exists within the calculated parameter space. At these positions more sophisticated radiative transfer models like PDR models probably lead to more consistent results. However, a comparison between LVG and PDR models in M 82 shows that constraints on $\mathrm{H}_{2}$ densities and beam-averaged column densities are very similar for both methods (Mao et al. 2000). Güsten et al. (1993) and Mao et al. (2000) concluded that in order to explain the observed line ratios, a two-component model of the molecular gas in M 82 is needed. But while models of Güsten et al. (1993) favor the existence of a warm, dense $\left(T_{\text {kin }}=50-70 \mathrm{~K} ; n\left(\mathrm{H}_{2}\right)=10^{5} \mathrm{~cm}^{-3}\right)$ and a cold, diffuse $\left(T_{\text {kin }}=20-30 \mathrm{~K} ; n\left(\mathrm{H}_{2}\right)=10^{3} \mathrm{~cm}^{-3}\right)$ gas component, Mao et al. (2000) find that the bulk of CO emission arises from a warm, diffuse component. Our high-resolution LVG results confirm the PDR calculations of Mao et al. (2000). In particular, their conclusion that the bulk of $\mathrm{CO}$ emission in the core of M 82 arises from a warm, low-density interclump medium is consistent with our findings.

Further support for a highly excited $\mathrm{CO}$ component towards the active regions in M 82 comes from the morphology of the high- $J$ CO lines observed by Mao et al. (2000). They find that the spatial separation of the CO lobes decreases with increasing $J$. While the spatial separation of the outer $\mathrm{CO}$ lobes in the ${ }^{12} \mathrm{CO}(J=1 \rightarrow 0)$ and ${ }^{12} \mathrm{CO}(J=2 \rightarrow 1)$ transition is about $26^{\prime \prime}$, it decreases to only $15^{\prime \prime}$ in the ${ }^{12} \mathrm{CO}(J=7 \rightarrow 6)$ transition. This distance is in good agreement with the spatial separation of the kinetic temperature peaks that we find in our LVG solutions (Fig. 8a). 


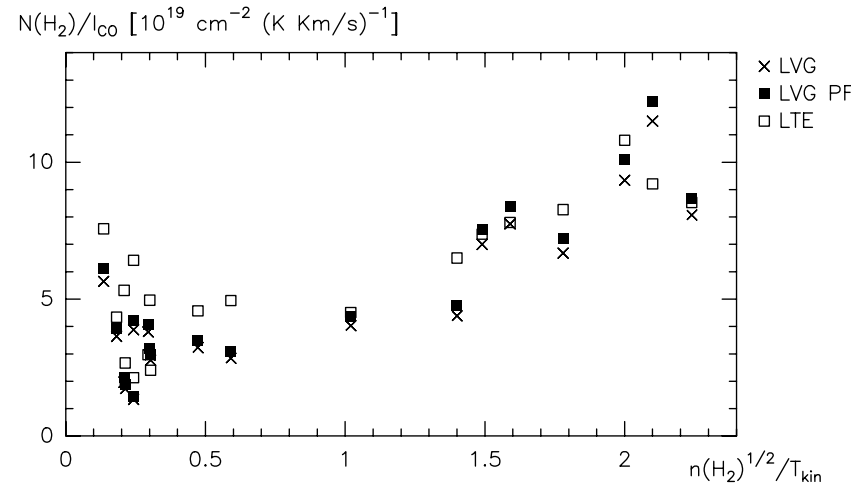

Fig. 11. $X_{\mathrm{CO}}$ as derived from the LTE, LVG and LVGPF solutions versus $T^{-1} n\left(\mathrm{H}_{2}\right)^{1 / 2}$. The plot shows a linear correlation between the conversion factor and the associated $\mathrm{H}_{2}$ densities and kinetic temperatures beyond $T^{-1} n\left(\mathrm{H}_{2}\right)^{1 / 2} \approx 0.5$

To further test the reliability of the modeled kinetic temperature and $\mathrm{H}_{2}$ density distribution across M 82 we calculated line ratios for the high- $J$ transitions of ${ }^{12} \mathrm{CO}$ and ${ }^{13} \mathrm{CO}$ at $22^{\prime \prime}$ resolution and compared our prediction with the line ratios published by Mao et al. (2000). Note that the spatial smoothing of our high-resolution onecomponent excitation model leads to a multi-component model at lower resolution because it encompasses the individual solutions (weighted with a Gaussian of $22^{\prime \prime}$ width) at all positions. The predicted line ratios from a single CO isotope match the observations extremely well. For different $\mathrm{CO}$ isotopes the predicted line ratios are lower than suggested by the observations, but consistent within the observational errors. The predicted and observed line ratios are summarized in Table 3.

An independent chain of arguments favoring a temperature gradient towards the central starburst region comes from the different separation of the eastern and western "hot spots" in MIR and FIR observations. Hughes et al. (1994) stated that the larger separation of the peaks at $450 \mu \mathrm{m}$ reflects the radial temperature gradient that must exist within the torus if the dust is heated by the central starburst population.

\subsection{The state of the molecular gas in M82's center}

Our study does not imply that the central part of M 82 does not contain dense molecular cloud cores as traced by other molecular species like HCN (see e.g. Seaquist et al. 1985; Brouillet \& Schilke 1993). Most of the CO emission from the core of M 82, however, arises from a warm, low density interclump medium. This conclusion was already reached by Mao et al. (2000) using PDR models. The existence of such a gas component, which we can be confident of, raises the question if it can survive under the extreme conditions in the starburst center or whether it is indicative of cloud evaporation and thus of a destruction of molecular clouds. From the observed UV field
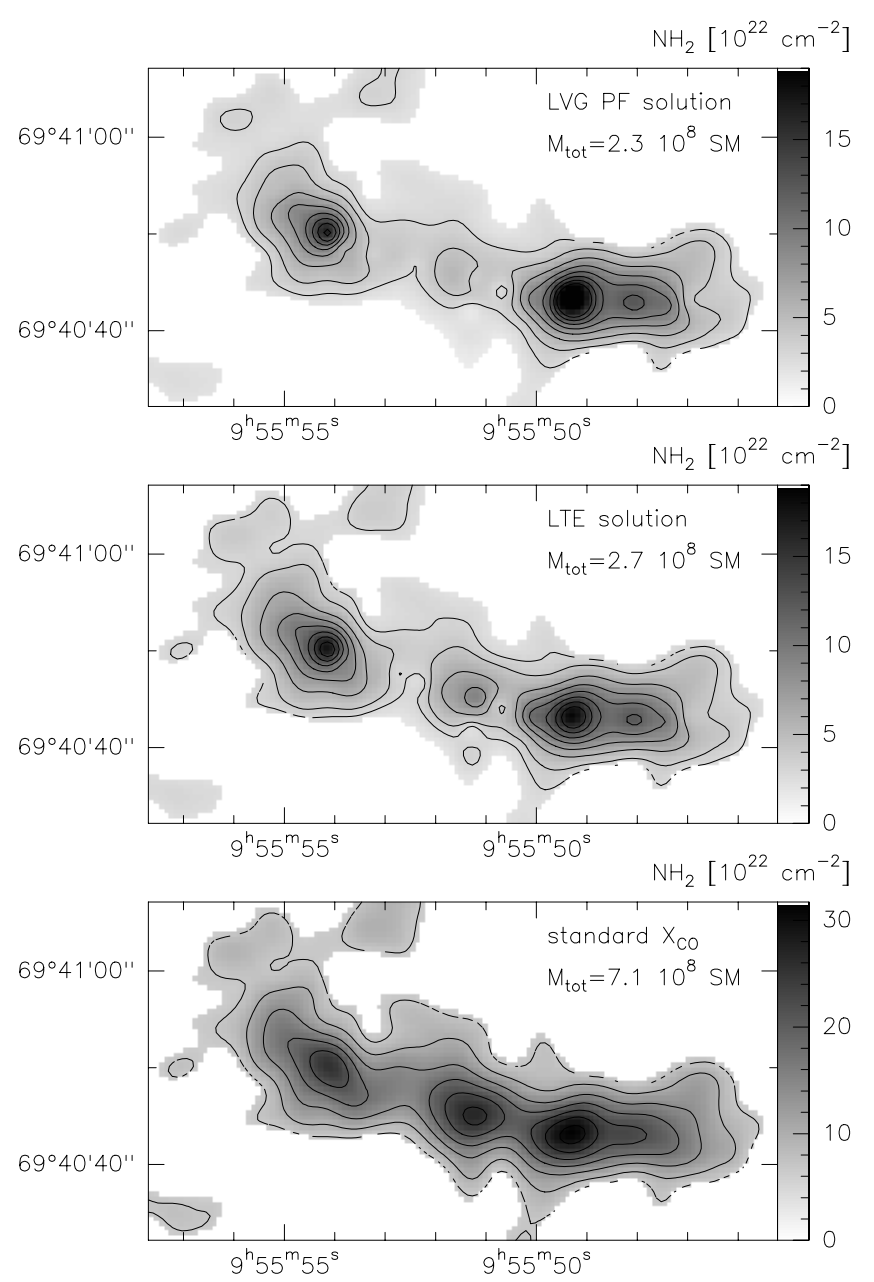

Fig. 12. $\mathrm{H}_{2}$ column density maps calculated from the integrated ${ }^{12} \mathrm{CO}(J=1 \rightarrow 0)$ intensity distribution at $4.2^{\prime \prime}$ resolution. The $\mathrm{H}_{2}$ maps were derived using the $X_{\mathrm{CO}}$ distribution across the major axis of $\mathrm{M} 82$ as calculated from the LVG $_{\mathrm{PF}}$ solutions (top), from the LTE solutions (middle) and from the standard Galactic $X_{\mathrm{CO}}$ conversion factor (1.6 $\left.10^{20} \mathrm{~cm}^{-2}\left(\mathrm{~K} \mathrm{~km} \mathrm{~s}^{-1}\right)^{-1}\right)$ (bottom). The total $\mathrm{H}_{2}$ mass is denoted in the upper right corner of each plot. Contour levels correspond to $1.4,2,3,4,6,8,10,12,14$ and $1610^{22} \mathrm{~cm}^{-2}$ (top), 3, 4, 6, 8, 10, 12, 14 and $1610^{22} \mathrm{~cm}^{-2}$ (middle) and 7 , $10,14,18,22,26$ and $3010^{22} \mathrm{~cm}^{-2}$ (bottom)

strength one would expect that the molecular clouds with densities less than $10^{4} \mathrm{~cm}^{-3}$ cannot exist in M 82 's center (Brouillet \& Schilke 1993). In addition, such a gas component is barely dense enough to avoid tidal disruption (Mao et al. 2000) which would support a rather diffuse distribution of the gas. Such a scenario would explain the high $[\mathrm{CI}] /[\mathrm{CO}]$ abundance ratios observed in M 82 (Schilke et al. 1993; White et al. 1994) and the depletion of $\mathrm{H}_{2}$ in the central $300 \mathrm{pc}$ of M 82 . As a consequence, this would imply that the clouds are not virialized. This could explain why the linear correlation between $T_{\mathrm{kin}}^{-1} n\left(\mathrm{H}_{2}\right)^{1 / 2}$ and $X_{\mathrm{CO}}$ is not valid for $T_{\mathrm{kin}}^{-1} n\left(\mathrm{H}_{2}\right)^{1 / 2}<0.5$, hence for the warm and low-density gas in the starburst center (see Fig. 11). Therefore, there seems to be observational evidence that 
Table 3. Line ratios for the high- $J$ transition of ${ }^{12} \mathrm{CO}$ and ${ }^{13} \mathrm{CO}$ at the center and the eastern and western $\mathrm{CO}$ lobe of M 82. The line ratios are given for a spatial resolution of $22^{\prime \prime}$. The first row of each line ratio gives the value calculated from the LVG model. The second and third row are the observed peak and integrated line ratios adopted from Mao et al. (2000)

\begin{tabular}{lcccl}
\hline CO line ratio & east & center & west & \\
\hline${ }^{12} \mathrm{CO}(7 \rightarrow 6) /{ }^{12} \mathrm{CO}(4 \rightarrow 3)$ & 0.36 & 0.25 & 0.31 & model \\
& 0.32 & 0.29 & 0.37 & peak \\
& 0.28 & 0.36 & 0.33 & integral \\
& & & & \\
${ }^{12} \mathrm{CO}(4 \rightarrow 3) /{ }^{13} \mathrm{CO}(3 \rightarrow 2)$ & 7.07 & 8.02 & 5.81 & model \\
& 8.50 & 10.1 & 9.07 & peak \\
& 9.09 & 6.88 & 7.40 & integral \\
& & & & \\
${ }^{13} \mathrm{CO}(2 \rightarrow 1) /{ }^{13} \mathrm{CO}(1 \rightarrow 0)$ & 1.92 & 1.67 & 1.87 & model \\
& 1.53 & 1.63 & 1.93 & peak \\
& 1.47 & 1.44 & 1.17 & integral \\
& & & & \\
${ }^{12} \mathrm{CO}(2 \rightarrow 1) /{ }^{13} \mathrm{CO}(2 \rightarrow 1)$ & 7.82 & 9.27 & 7.48 & model \\
& 9.04 & 13.7 & 9.19 & peak \\
& 11.4 & 14.2 & 11.7 & integral \\
\hline
\end{tabular}

molecular clouds are partly disrupted and dissociated in the starburst center. More detailed numerical analysis of evaporation time scales and related questions are needed to settle this question.

\section{3. $X_{\mathrm{CO}}$}

The suitability of a global Galactic factor $X_{\mathrm{CO}}$ to convert ${ }^{12} \mathrm{CO}(J=1 \rightarrow 0)$ intensities to $\mathrm{H}_{2}$ column densities has been discussed by many authors (e.g. Young \& Scoville 1982; Bloemen et al. 1986; Hunter et al. 1997). As seen, theoretical studies of $X_{\mathrm{CO}}$ showed that it is sensitive to the kinetic temperature of the emitting gas and that the conversion factor should be lower for starburst galaxies like M 82 than for the Milky Way (Maloney \& Black 1988). Investigations of $X_{\mathrm{CO}}$ in M 82 confirmed this prediction: Wild et al. (1992) used the optically thin $\mathrm{C}^{18} \mathrm{O}(2 \rightarrow 1)$ transition to derive $\mathrm{H}_{2}$ column densities and hence $X_{\mathrm{CO}}$ along the major axis of M 82. They found $X_{\mathrm{CO}} \leq 110^{20} \mathrm{~cm}^{-2}\left(\mathrm{~K} \mathrm{~km} \mathrm{~s}^{-1}\right)^{-1}$ and variations by a factor of 2 along the major axis. Similar results were obtained by Smith et al. (1991) using the $450 \mu \mathrm{m}$ continuum emission from dust grains to derive $\mathrm{H}_{2}$ column densities. Even though both studies suggest a low conversion factor, its variation across the major axis shows significant differences. While Smith et al. (1991) found that $X_{\mathrm{CO}}$ decreases from east to west, with no changes in the central starforming regions, the results by Wild et al. (1992) suggest very low conversion factors near the eastern MIR peak and an increasing $X_{\mathrm{CO}}$ towards the western molecular lobe. Therefore our variations of $X_{\mathrm{CO}}$ in general support the results by Wild et al. (1992). Nevertheless our conversion factors are slightly lower than those inferred by Wild et al. (1992), and the location of the western $X_{\mathrm{CO}}$ maximum is displaced by $\approx 7^{\prime \prime}$. The discrepancies between the $X_{\mathrm{CO}}$ factors derived from the molecular lines (Wild et al. 1992 and this work) and the estimates from the dust emission might result from the single-temperature model used by Smith et al. (1991). In particular in the central region, which shows strong MIR emission from heated dust (Telesco \& Gezari 1992), this might lead to an overestimate of the $\mathrm{H}_{2}$ column density and thus to an overestimate of $X_{\mathrm{CO}}$. Furthermore, the different morphology of the $450 \mu \mathrm{m}$ map published by Hughes et al. (1994) raises doubts on the reliability of the $450 \mu \mathrm{m}$ intensities used by Smith et al. (1991) for their calculation. From this we conclude that the $X_{\mathrm{CO}}$ factor in M 82 is not only lower than the standard Galactic conversion factor, but that in addition $X_{\mathrm{CO}}$ in the central $300 \mathrm{pc}$ is at least 3 times lower than in the molecular lobes. A similar gradient for the conversion factor has been found in the Milky Way towards the Galactic Center (e.g. Blitz et al. 1985; Sodroski et al. 1994; Dahmen et al. 1998). Furthermore, our analysis suggests that the variations of $X_{\mathrm{CO}}$ are mainly caused by variations of the kinetic temperature of the $\mathrm{CO}$-emitting volume due to environmental effects while abundance variations play a minor role.

\section{Conclusions}

We have observed the ${ }^{12} \mathrm{CO}(J=2 \rightarrow 1)$ and $\mathrm{C}^{18} \mathrm{O}(J=1 \rightarrow 0)$ emission lines in the starburst galaxy M82 with high spatial resolution using the Plateau de Bure interferometer. Our main conclusions are:

1) The overall morphology and kinematics for both transitions are similar to those of ${ }^{12} \mathrm{CO}(J=1 \rightarrow 0)$ and ${ }^{13} \mathrm{CO}(J=1 \rightarrow 0)$ published by Shen \& Lo (1995) and Neininger et al. (1998). The dynamical center of the molecular gas coincides with the $2.2 \mu \mathrm{m}$ nucleus while the centroid of the molecular mass is located $100 \mathrm{pc}$ west of M 82's center. South of the expanding molecular superbubble (Weiß et al. 1999) an outflow of molecular gas with a total mass of $7.210^{5} M_{\odot}$ is detected;

2) The ${ }^{12} \mathrm{CO}(J=2 \rightarrow 1) /{ }^{12} \mathrm{CO}(J=1 \rightarrow 0)$ line intensity ratios are lower $(\leq 1.4)$ than previously reported. Thus, CO line ratios in M82 are not outstanding, but comparable to values found in other starburst galaxies like NGC 253. Line ratios vary across the disk of M 82. Near the MIR peaks, the ${ }^{12} \mathrm{CO}(J=2 \rightarrow 1) /{ }^{12} \mathrm{CO}(J=1 \rightarrow 0)$ 
ratios are high; in the outer parts, that are less affected by the starburst, this ratio drops to unity;

3) An LVG excitation analysis of the CO lines suggests that the excitation conditions of the molecular gas are strongly influenced by environmental effects. In the outer parts of the $\mathrm{CO}$ distribution we find $\mathrm{H}_{2}$ densities of $n\left(\mathrm{H}_{2}\right)=10^{3.5-4.2} \mathrm{~cm}^{-3}$ and kinetic temperatures of $T_{\text {kin }}=45-60 \mathrm{~K}$. Towards the star-forming regions, indicated by strong MIR emission, the kinetic temperatures raise above $150 \mathrm{~K}$. The hot gas is associated with low $\mathrm{H}_{2}$ densities of only $n\left(\mathrm{H}_{2}\right)=10^{2.8-3.1} \mathrm{~cm}^{-3}$. Area filling factors of $f_{\mathrm{a}}=0.2-0.4$ and volume filling factors of $f_{\mathrm{v}}=0.001-0.02$ indicate that the gas is organized in small clumps with a typical size of $r_{\text {cloud }} \approx 1$ pc. $\left[{ }^{12} \mathrm{CO}\right] /\left[{ }^{13} \mathrm{CO}\right]$ abundance ratios are about 70 without significant spatial variations across the galaxy. In contrast, $\left[{ }^{12} \mathrm{CO}\right] /\left[\mathrm{C}^{18} \mathrm{O}\right]$ abundance ratios in the outer parts of $\mathrm{M} 82$ are comparable to those found at the Galactic Center $\left(\left[{ }^{12} \mathrm{CO}\right] /\left[\mathrm{C}^{18} \mathrm{O}\right]=270\right)$ but decrease to only $\left[{ }^{12} \mathrm{CO}\right] /\left[\mathrm{C}^{18} \mathrm{O}\right]=160$ at the star-forming regions. Beam-averaged $\mathrm{H}_{2}$ column densities range from $N\left(\mathrm{H}_{2}\right)_{4^{\prime \prime}}=2.410^{22} \mathrm{~cm}^{-2}$ near the MIR peaks to $N\left(\mathrm{H}_{2}\right)_{4^{\prime \prime}}=2.310^{23} \mathrm{~cm}^{-2}$ at the western CO lobe. The $\mathrm{H}_{2}$ distribution has a double-peak morphology which surrounds the central starburst region. The central $300 \mathrm{pc}$ are depleted in $\mathrm{H}_{2}$. Thus the $\mathrm{H}_{2}$ distribution differs from the $\mathrm{CO}$ distribution. This result even holds when the $\mathrm{H}_{2}$ column densities are calculated under the assumption of LTE conditions. The total molecular mass is $2.310^{8} M_{\odot}$;

4) The conversion factor from $I(\mathrm{CO})$ to $N\left(\mathrm{H}_{2}\right)\left(X_{\mathrm{CO}}\right)$ depends on the excitation conditions of the $\mathrm{CO}$-emitting volume. Even in regions which are less affected by the starburst $X_{\mathrm{CO}}$ is about 3 times lower than the standard Galactic value. From the LVG analysis we find that $X_{\mathrm{CO}} \sim T_{\text {kin }}^{-1} n\left(\mathrm{H}_{2}\right)^{1 / 2}$. Therefore $X_{\mathrm{CO}}$ is lower in the central star-forming regions than in the outer molecular lobes.

\section{Appendix: Short-spacing correction}

A major difficulty related to flux determinations from interferometer maps is the missing flux problem. In brief it arises from the lack of coverage at low spatial frequencies in interferometric observations. This leads to an insensitivity to emission more extended than $\lambda / S_{\mathrm{min}}^{\mathrm{proj}}$, where $S_{\mathrm{min}}^{\mathrm{proj}}$ is the length of the shortest projected baseline. The recovered flux therefore only represents clumpy parts of a brightness distribution which leads to an underestimate of the total flux of a source. For a more detailed description see e.g. Wilner \& Welch (1994) and Helfer \& Blitz (1995). The only way to overcome this problem is to derive the visibility at the origin of the $u v$-plane (which represents the integrated flux of a brightness distribution) and the low spatial frequencies from single-dish measure- ments and combine them with the interferometric observations. Methods for the combination of single-dish and interferometer data have been described e.g. by Vogel et al. (1984) and Herbstmeier et al. (1996). Both methods generate the central visibilities from the single-dish data, as if they were measured with the interferometer. The combined visibility set is then processed in the standard interferometer reduction procedure. In this method the relative weight between single-dish and interferometer visibilities has a strong effect on the resulting brightness distribution. Furthermore the dirty image calculated from the combined visibility set still needs to be deconvolved using CLEAN or other deconvolution algorithms. In particular the CLEAN deconvolution algorithm is problematic for short-spacing corrected images because it fails on extended structures. We therefore used a different approach to combine the PdBI and $30 \mathrm{~m}$ telescope data.

The basic idea behind our method is that the missing flux problem only arises from an incorrect interpolation of the visibilities in the central part of the $u v$-plane $\left(\sqrt{u^{2}+v^{2}}<\lambda / S_{\min }^{\mathrm{proj}}\right)$. Therefore the missing flux problem in a finally reduced interferometer map can be solved by replacing the questionable part of the $u v$-plane by the values calculated from a single-dish map with identical extent, grid and flux units. This procedure avoids additional CLEANing on the combined data set and the choice of different weightings between interferometer and singledish data. The requirement for the single-dish data is the same as described by Vogel et al. (1984). For the combination we regridded the single-dish data cube to the same spatial and velocity grid as the interferometer data. We then converted the flux units from $T_{\mathrm{mb}}$ to Jy/pixel using the Rayleigh-Jeans approximation, $S /$ beam $=\frac{2 k}{\lambda^{2}} T_{\mathrm{mb}}$ and beam $=1.133 f_{\mathrm{s}}^{2}$ where $f_{\mathrm{s}}$ is the $F W H M$ of the $30 \mathrm{~m}$ telescope beam in units of the pixel size. The flux units of the CLEANed and primary beam corrected interferometer cube were also converted to Jy/pixel with beam $=1.133 f_{i, \text { maj }} \cdot f_{i, \text { min }}$, where $f_{i, \text { maj }}$ and $f_{i, \text { min }}$ are the FWHM of the major and minor axis of the clean beam in units of the pixel size. Furthermore we generated a model for the $30 \mathrm{~m}$ telescope main beam and the interferometer clean beam. The $30 \mathrm{~m}$ beam was assumed to be represented by a circular Gaussian with $F W H M=$ $f_{\mathrm{s}}$. The interferometric beam was described as a Gaussian with major and minor axis $f_{i \text {,maj }}$ and $f_{i, \text { min }}$. The normalization of both Gaussians was such that the amplitude of the visibility at the origin of the $u v$-plane was 1 . We then transformed both data cubes and the model beams to the $u v$-domain using an FFT algorithm. The real and imaginary parts of the single-dish data were divided by the amplitudes of the model $30 \mathrm{~m}$ beam to deconvolve the single-dish visibilities from the $30 \mathrm{~m}$ telescope beam. In order to match the interferometer data the result was then multiplied by the amplitudes of the clean beam. At this stage of the combination the real and imaginary parts in the single-dish and interferometer data are comparable and the central interferometer pixels can be replaced by the single-dish values. The part of the $u v$-domain to be 


\section{Short-Spacing Correction}

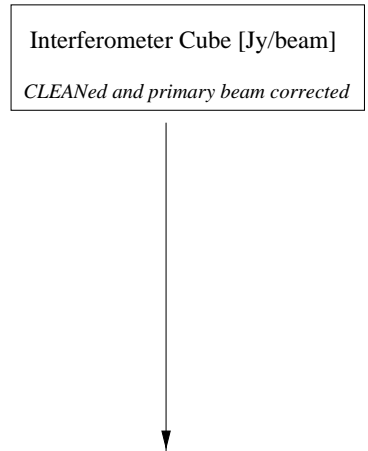

Flux conversion to Jy/pixel

$1 \mathrm{Jy} /$ beam $=1.133$ bmaj $i *$ bmin $i /$ pixel

1

Interferometer Cube [Jy/pixel]

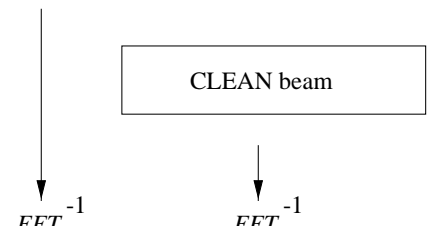

$F F T^{-1}-F F T^{-1}$

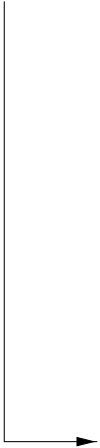

Replace inner visibilities of

interferometer data with

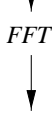

Flux conversion to Jy/beam

$1 \mathrm{Jy} / \mathrm{b}=0.883 \mathrm{pixel} /(\mathrm{bmaj}, i * \mathrm{bmin}, \mathrm{i}) \quad \mathrm{Jy} / \mathrm{pixel}$

SSC Cube [Jy/beam] single-dish values

Single-dish Cube $[\mathrm{K}]$

Flux units in Tmb

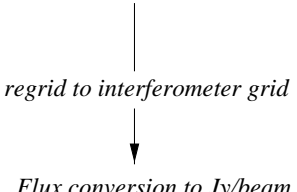

Flux conversion to Jy/beam

$1 K=7.353 * 10^{-08} \lambda^{-2}[\mathrm{~m}] \quad$ bmaj $*$ bmin $\left[{ }^{\prime \prime}\right] \quad \mathrm{Jy} / \mathrm{b}$

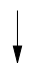

Flux conversion to Jy/pixel

$1 \mathrm{Jy} /$ beam $=1.133$ bmaj,s $*$ bmin,s $/$ pixel $\quad$ Jy/pixel

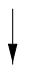

Single-dish Cube [Jy/pixel]

Model Single-dish beam
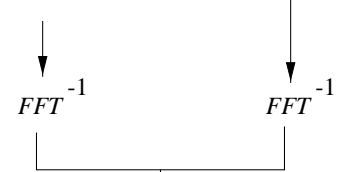

Deconvolution

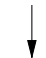

Convolution with clean beam

Fig. 13. Flow chart of the short-spacing correction 
replaced by the single--dish values in general depends on the spacing of the shortest baseline and on the effective diameter of the single-dish telescope. For data sets with no overlap in the $u v$-domain we replaced the part that corresponds to the effective diameter of the $30 \mathrm{~m}$ telescope (amplitude of the visibilities for the $30 \mathrm{~m}$ beam model $>0.5$ ). Otherwise we selected the part smaller than the shortest interferometer baseline. Finally the combined real and imaginary parts were transformed back to the image domain and the flux units were converted to Kelvin considering that the combined beam is equal to the clean beam.

Note that there are no free parameters in the combination of the data sets except for the choice which part of the $u v$-plane is replaced by the single-dish data. The methods require the knowledge of the single-dish beam pattern and the clean beam only. A flow chart for the Short-Spacing correction is given in Fig. 13.

Acknowledgements. We with to thank the IRAM staff for carrying out the observations and the help provided during the data reduction. We thank J. Shen and K. Y. Lo for making available their CO data and C. Henkel and A. Heithausen for many fruitful discussions. This research has been supported by the Deutsche Forschungsgemeinschaft (DFG) though grant III GK-GRK 118/2.

\section{References}

Achtermann, J. M., \& Lacy, J. H. 1995, ApJ, 439, 163

Bland, J., \& Tully, B. 1988, Nat, 334, 43B

Blake, G. A., Sutton, E. C., Masson, C. R., \& Phillips, T. G. 1987, ApJ, 315, 621

Blitz, L., Bloemen, J. B. G. M., Hermsen, W., \& Bania, T. M. 1985, A\&A, 143, 267

Bloemen, J. B. G. M., Strong, M. W., Mayer-Hasselwander, H. A., et al. 1986, A\&A, 154, 25

Bregman, J. N., Schulman, E., \& Tomisaka, K. 1995, ApJ, 439, 155

Brouillet, N., \& Schilke, P. 1993, A\&A, 277, 381

Dahmen, G., Hüttemeister, S., Wilson, T. L., \& Mauersberger, R. 1998, A\&A, 331, 959

de Jong, T., Chu, S. I., \& Dalgarno, A. 1975, ApJ, 199, 69

Dickman, R. L. 1978, ApJS, 37, 407

Dietz, K., Smith, J., Hackwell, J. A., Gehrz, D. R., \& Grasdalen, G. L. 1986, AJ, 91, 758

Farquhar, P. R. A., Millar, T. J., \& Herbst, E. 1994, MNRAS, 269,641

Goldreich, J., \& Kwan, J. 1974, ApJ, 191, 93

Greve, A., Kramer, C., \& Wild, W. 1998, A\&AS, 133, 271

Guélin, M., Kramer, C., \& Wild, W. 1995, IRAM Newsletter, 19,17

Güsten, R., Serabyn, E., Kasemann, C., et al. 1993, A\&A, 402, 537

Helfer, T. T., \& Blitz, L. 1995, ApJ, 450, 90
Henkel, C., \& Bally, J. 1985, A\&A, 150, L25

Henkel, C., Chin, Y.-N., Mauersberger, R., \& Whiteoak, J. B. 1998, A\&A, 329, 443

Herbstmeier, U., Kalberla, P. M. W., \& Mebold, U. 1996, A\&AS, 117, 497

Hughes, D. H., Gear, W. K., \& Robson, E. I. 1994, MNRAS, 270,641

Hunter, S. D., Bertsch, D. L., Catelli, J. R., et al. 1997, ApJ, 481, 205

Knapp, G. R., Leighton, R. B., Wannier, P. G., Phillips, T. G., \& Huggins, P. J. 1980, ApJ, 240, 60

Kronberg, P. P., Biermann, P., \& Schwab, F. R. 1985, ApJ, 291, 693

Loiseau, N., Nakai, N., Wielebinski, R., Reuter, H.-P., \& Klein, U. 1990, A\&A, 228, 331

Maloney, P., \& Black, J. H. 1988, ApJ, 325, 389

Mao, R. Q., Henkel, C., Schulz, A., et al. 2000, A\&A, 358, 433

McKeith, C. D., Greve, A., Downes, D., \& Prada, F. 1995, A\&A, 293, 703

Nakai, N., Hayashi, M., Handa, T., Sofue, Y., \& Hasegawa, T. 1987, PASJ, 39, 685

Neininger, N., Guélin, M., Klein, U., García-Burillo, S., \& Wielebinski, R. 1998, A\&A, 339, 737

Olofsson, H., \& Rydbeck, G. 1984, A\&A, 136, 17

Petitpas, G. R., \& Wilson, C. D. 2000, ApJ, 538, 117

Rieke, G. H., Lebofsky, M. J., Thompson, R. I., Low, F. J., \& Tokunaga, A. T. 1980, ApJ, 238, 24

Sakai, S., \& Madore, B. F. 1999, ApJ, 526, 599

Schilke, P., Carlstrom, J. E., Keene, J., \& Phillips, T. G. 1993, ApJ, 417, L97

Seaquist, E. R., Bell, M. B., \& Bignell, R. C. 1985, ApJ, 294, 546

Shen, J., \& Lo, K. Y. 1995, ApJ, 445, L99

Shopbell, P. L., \& Bland-Hawthorn, J. 1998, ApJ, 493, 129

Smith, P. A., Brand, P. W. J. L., Mountain, C. M., Puxley, P. J., \& Nakai, N. 1991, MNRAS, 252, L6

Sodroski, T. J., Bennett, C., Boggess, N., et al. 1994, ApJ, 428, 638

Tammann, G. A., \& Sandage, A. 1968, ApJ, 151, 825; 1991, ApJ, 369, 135

Telesco, C. M., \& Gezari, D. Y. 1992, ApJ, 395, 461

Tilanus, R. P. J., Tacconi, L. J., Sutton, E. C., Lo, K. Y., \& Stephens, S. A. 1991, ApJ, 376, 500

Vogel, S. N., Wright, M. C. H., Plambeck, R. L., \& Welch, W. J. 1984, ApJ, 283, 655

Weiß, A., Walter, F., Neininger, N., \& Klein, U. 1999, A\&A, 345, L23

White, G. J., Ellison, B., Claude, S., Dent, W. R. F., \& Matheson, D. N. 1994, A\&A, 284, L23

Wilner, D. J., \& Welch, W. J. 1994, ApJ, 427, 898

Wild, W., Harris, A. I., Eckart, A., et al. 1992, A\&A, 265, 447

Wills, K. A., Redman, M. P., Muxlow, T. W. B., \& Pedlar, A. 1999, MNRAS, 309, 395

Wolfire, M. G., Tielens, A. G. G. M., \& Hollenbach, D. 1990, ApJ, 358, 116

Young, J. S., \& Scoville, N. Z. 1982, ApJ, 258, 467 